\title{
WestVirginiaUniversity
}

THE RESEARCH REPOSITORY @ WVU

Graduate Theses, Dissertations, and Problem Reports

2008

\section{Performance and area optimization for reliable FPGA-based shifter design}

\author{
Zahid Ali Syed \\ West Virginia University
}

Follow this and additional works at: https://researchrepository.wvu.edu/etd

\section{Recommended Citation}

Syed, Zahid Ali, "Performance and area optimization for reliable FPGA-based shifter design" (2008). Graduate Theses, Dissertations, and Problem Reports. 1963.

https://researchrepository.wvu.edu/etd/1963

This Thesis is protected by copyright and/or related rights. It has been brought to you by the The Research Repository @ WVU with permission from the rights-holder(s). You are free to use this Thesis in any way that is permitted by the copyright and related rights legislation that applies to your use. For other uses you must obtain permission from the rights-holder(s) directly, unless additional rights are indicated by a Creative Commons license in the record and/ or on the work itself. This Thesis has been accepted for inclusion in WVU Graduate Theses, Dissertations, and Problem Reports collection by an authorized administrator of The Research Repository @ WVU. For more information, please contact researchrepository@mail.wvu.edu. 


\title{
Performance and Area Optimization for Reliable FPGA-based Shifter Design
}

\author{
by
}

\author{
Zahid Ali Syed \\ Thesis submitted to the \\ at West Virginia University \\ in partial fulfillment of the requirements \\ for the degree of \\ Master of Science \\ in \\ Electrical Engineering \\ Afzel Noore, Ph.D., Chair \\ James D. Mooney, Ph.D. \\ Hany H. Ammar, Ph.D.
} College of Engineering and Mineral Resources

Lane Department of Computer Science and Electrical Engineering

$$
\begin{gathered}
\text { Morgantown, West Virginia } \\
2008
\end{gathered}
$$

Keywords: FPGA, Reliable Shifter Design, Optimization, Assertion Based Verification, Synthesis, Open Verification Library, Embedded Logic

(C) Zahid Syed, 2008 


\begin{abstract}
Performance and Area Optimization for Reliable FPGA-based Shifter Design

by

Zahid Ali Syed

Master of Science in Electrical Engineering

West Virginia University

Afzel Noore, Ph.D., Chair
\end{abstract}

This thesis addresses the problem of implementing reliable FPGA-based shifters. An FPGA-based design requires optimization between performance and resource utilization, and an effective verification methodology to validate design behavior. The FPGA-based implementation of a large shifter design is restricted by an I/O resource bottleneck. The verification of the design behavior presents a further challenge due to the 'black-box' nature of FPGAs. To tackle these design challenges, we propose a novel approach to implement FPGA-based shifters. The proposed design alleviates the I/O bottleneck while significantly reducing the logic resources required. This is achieved with a minimal increase in the design delay. The design is seamlessly scalable to a multi-FPGA chip setup to improve performance or to implement larger shifters. It is configured using assertion checkers for efficient design verification. The assertion-based design is further optimized to alleviate the performance degradation caused by the assertion checkers. 


\section{Acknowledgements}

My stay at West Virginia University has been a period of constant learning. My teachers, friends and family have been a part of my graduate education and I would like to take this opportunity to express my gratitude towards them.

It has been an honor to have had Dr Noore as my mentor and research advisor. I have much to thank him for, from his untiring encouragement to his objective criticism. I am obliged to him for having tolerated my constant stream of questions, and unscheduled interruptions for advice. His support as a friend and guide has made me a better person in many aspects of life.

I would also like to thank Dr. Ammar and Dr. Mooney, my committee members, for their suggestions and guidance.

My thanks go out to my friends for their support and making my stay at WVU memorable: Ajit, Ashwin, Harry, Mangu, Nitin, Prasad, Praveen, Verma, Vijith, and the rest. You know who you are.

My family has been a pillar of support throughout my life. My parents and siblings have always offered the moral support necessary to perform well and I am indebted to them for standing by me in times of need. My wife has been the chief driving force behind the completion of this thesis.

And finally, I thank my cousin and roommate Ahad. Our stay at WVU has been fruitful in great part through our mutual support. There is little by way of words that might express my gratitude for this. 


\section{Table of Contents}

Acknowledgements. . . . . . . . . . . . . . . . . . . . . . . . iii

List of Figures. . . . . . . . . . . . . . . . . . . . . . . . . . . . . . vi

List of Tables. . . . . . . . . . . . . . . . . . . . . . . . . . . . . . vii

1 Introduction, Problem Statement and Methodology. . . . . . . . . 1

1.1 FPGAs and FPGA programming. . . . . . . . . . . . . . . . . . . 1

1.2 Assertion-based verification. . . . . . . . . . . . . . . . . . . . 2

1.3 Problem statement. . . . . . . . . . . . . . . . . . . . . . 3

1.4 Thesis organization. . . . . . . . . . . . . . . . . . . . . . 4

2 Literature survey. . . . . . . . . . . . . . . . . . . . . . . . . 5

2.1 Shifter design. . . . . . . . . . . . . . . . . . . . . . . . . . . . 5

2.2 Assertion-based verification. . . . . . . . . . . . . . . . . 6

3 Field Programmable Gate Arrays. . . . . . . . . . . . . . . . . . . 8

3.1 Introduction. . . . . . . . . . . . . . . . . . . . . 8

3.2 Uses. . . . . . . . . . . . . . . . . . . . . . 8

3.3 Manufacturers. . . . . . . . . . . . . . . . . . . 9

3.4 The Spartan-3A series. . . . . . . . . . . . . . . . . . . . 10

3.5 FPGA programming and design approach. . . . . . . . . . . . 10

3.6 HDL modeling. . . . . . . . . . . . . . . . . . . . . 12

4 Shifter Design and FPGA-based Shifter Limitations. . . . . . . . . . 14

4.1 Common shifter designs. . . . . . . . . . . . . . . . . . . . . . . 14

4.2 FPGA-based shifter limitations. . . . . . . . . . . . . . . 18

5 Proposed FPGA-based shifter design. . . . . . . . . . . . . . . . . 19

5.1 Proposed design of an embedded auxiliary shifter. . . . . . . . . . . 19

5.2 Sequential-logic level processing. . . . . . . . . . . . . . . 20

5.3 Embedded auxiliary shifter and shift-control partitioning. . . . . . . . 21

5.4 Combinational-logic level processing. . . . . . . . . . . . . . . 22

5.5 Index updating. . . . . . . . . . . . . . . . . . . . . . . 23

5.6 Design optimization. . . . . . . . . . . . . . . . . . . . . . . 23

5.7 Extension to multi-chip FPGA design. . . . . . . . . . . . . . . . 24

6 Implementation of Shifter Designs in HDL. . . . . . . . . . . . . . 26

6.1 Barrel shifter. . . . . . . . . . . . . . . . . . . . 26

6.2 Logarithmic shifter. . . . . . . . . . . . . . . . . 27

6.3 Shift register. . . . . . . . . . . . . . . . . . . . . 27

6.4 Proposed auxiliary shifter design. . . . . . . . . . . . . . . 28

6.4.1 Sequential_logic. . . . . . . . . . . . . . . . . 28 
6.4.2 Combinational_logic. . . . . . . . . . . . . . . 29

6.5 Experimental results. . . . . . . . . . . . . . . . . 30

7 The VHDL Open Verification Library. . . . . . . . . . . . . . . . . 32

7.1 Controllability. . . . . . . . . . . . . . . . . . . 32

7.2 Observability. . . . . . . . . . . . . . . . . . 32

7.3 Challenges during verification. . . . . . . . . . . . . . . 33

7.4 Assertion-based verification . . . . . . . . . . . . . . . . 34

7.5 Assertion checker standards. . . . . . . . . . . . . . . . 35

7.6 Current assertion standards. . . . . . . . . . . . . . . . 36

7.6.1 Open Verification Library (OVL). . . . . . . . . . . . . . 36

7.6.2 X/Z Check in OVL checkers. . . . . . . . . . . . . . . . . 37

7.7 Performance characteristics of the VHDL OVL checkers. . . . . . . . 38

7.8 Assertions in the proposed shifter design. . . . . . . . . . . . . . . 39

7.9 Estimated resource overhead and performance reduction. . . . . . . . 41

8 Alleviating Performance Overhead Due to Assertions. . . . . . . . . 42

8.1 Partitioning and component removal in Xilinx ISE . . . . . . . . . . 43

8.1.1 Routing. . . . . . . . . . . . . . . . . . 44

8.1.2 Placement. .................... . . . 44

8.1.3 Synthesis. . . . . . . . . . . . . . . . . 44

8.1.4 Inherit. . . . . . . . . . . . . . . . . . . . . 45

8.2 Using Xilinx Partitions in the design under study. . . . . . . . . . . 45

8.3 Experimental results and analysis. . . . . . . . . . . . . . 45

8.3.1 Assertion-based design. . . . . . . . . . . . . . . . . 45

8.3.2 Re-configured assertion-free design. . . . . . . . . . . 48

9 Conclusion and Future Work. . . . . . . . . . . . . . . . . . . . 50

9.1 Conclusion. . . . . . . . . . . . . . . . . . 50

9.2 Future work. . . . . . . . . . . . . . . . 50

References. . . . . . . . . . . . . . . . . . . . . . . . 52

Appendix. . . . . . . . . . . . . . . . . . . . . . . . . . . . . . . . 54

Appendix A. . . . . . . . . . . . . . . . . . . . . . . 54

A.1 The VHDL OVL library . . . . . . . . . . . . . . . 54

A.1.1 The ovl_ctrl_record type. . . . . . . . . . . . . . . 54

A.1.2 Synthesizing the VHDL OVL library. . . . . . . . . . . . . . 55

Appendix B. . . . . . . . . . . . . . . . . . . . . 57 


\section{List of Figures}

3.1 EDA Design Flow. . . . . . . . . . . . . . . . . . . . . . . . 11

4.1 Design of a 4-bit Barrel Switch. . . . . . . . . . . . . . . . . . . 14

4.2 Design and working of a 4-bit barrel shifter. . . . . . . . . . . . . . 15

4.3 Design of an 8-bit logarithmic shifter. . . . . . . . . . . . . . . . 16

4.4 Design of an 8-bit logarithmic shifter/rotator. . . . . . . . . . . . . . . 17

5.1 Design of a 2048-bit shifter using 128-bit embedded logic. . . . . . . . . . 20

5.2 Control flow of the combinational logic. . . . . . . . . . . . . . . . 21

6.1 HDL design of a barrel shifter. . . . . . . . . . . . . . . . . . . . . 26

6.2 HDL Design of a logarithmic shifter. . . . . . . . . . . . . . . . . . 27

6.3 HDL hierarchy of the auxiliary shifter. . . . . . . . . . . . . . . 28 


\section{List of Tables}

3.1 Characteristics of the Spartan-3 Series. . . . . . . . . . . . . . . . . . 9

3.1 Characteristics of the Spartan-3A family. . . . . . . . . . . . . . . . . 10

6.1 Comparing performance metrics of the proposed FPGA approach with existing shifter designs. . . . . . . . . . . . . . . . . . . . 30

7.1 Currently available OVL Checkers. . . . . . . . . . . . . . . . . . . 37

7.2 OVL checker characteristics for the 64-bit embedded logic. . . . . . . . . 40

7.3 OVL checker characteristics for the 128-bit embedded logic. . . . . . . . . 40

7.4 Estimated performance characteristics of an assertion-based shifter design. . $\quad 41$

8.1 Performance characteristics of various assertion-based designs. . . . . . . . 47

8.2 Performance degradation in an assertion-based design compared to an assertion-free design. . . . . . . . . . . . . . . . . . . . . 47

8.3 Percentage error in estimating the performance metrics of an assertion-based design. . . . . . . . . . . . . . . . . . . . 47

8.4 Performance gain through assertion removal. . . . . . . . . . . . . . . 48

A.1 Description of the VHDL OVL checkers. . . . . . . . . . . . . . . . . 55 


\section{Chapter 1}

\section{Introduction, Problem Statement and Methodology}

Data shifting is an important operation of arithmetic processors, and array processors used for graphics and video processing applications. The operation is performed through shifters implemented using combinational logic or sequential logic. Each design approach has specific advantages that are useful in different applications. Barrel shifters and logarithmic shifters are combinational-logic based shifters that perform large shifts in a single clock cycle and are used in applications where speed is of primary importance. Sequential shift registers perform a single-bit shift every clock cycle but utilize fewer resources than combinational-logic based shifters. The synchronous design of the shift register also eliminates the possibility of the occurrence of a race condition in the circuit.

\subsection{FPGAs and FPGA programming}

Field Programmable Gate Arrays (FPGAs) are soft core processors and can be modified on the field unlike hard-wired processors. This makes FPGAs suitable for applications where the function of the chip may vary. Thus, the vendor manufactures the chip and the buyer configures it as needed. FPGAs are used in digital signal processors, DSP, software-defined radio, aerospace and defense systems, ASIC prototyping, medical imaging, computer vision, speech recognition, cryptography, bioinformatics, computer hardware emulation and a growing range of other areas. 
The logic for an FPGA-based design is commonly described using Hardware Description Language (HDL) tools. An HDL is used to formally describe the design and operation of electronic circuits, and to verify its operation by means of simulation. Examples of HDLs include SystemC, Verilog, VHDL, and SystemVerilog.

HDLs are thus used to create a design description for the required logic. The HDL design is then simulated and verified for proper functioning. The verification process is performed through various verification methodologies. The design is then synthesized for implementation on an FPGA using FPGA-design tools.

\subsection{Assertion-based verification}

Verification of a hardware design has become the biggest bottleneck in the time-tomarket of an FPGA design. Due to the increasing complexity of modern integrated circuits, verification requires up to $70 \%$ of the design time [14]. Any bug that is detected and fixed in a design may lead to further complications that have to be detected and fixed yet again. Due to this, there is a need for an efficient verification system that detects faults early in the design phase.

Assertion Based Verification (ABV) is a new verification methodology that offers several advantages over traditional verification. Assertions are, at the most basic level, logical expression checkers that return a binary value. In $\mathrm{ABV}$, assertion checks are inserted at strategic points in the code. This enables the design engineer to gain additional information on the state of the system during the simulation or verification process. Any internal error that occurs in the system is conveyed to the design engineer by these assertion monitors. In $\mathrm{ABV}$ methodology, the assertion checkers used to monitor the system extend beyond simple logical expression checking monitors. For example, a checker may be assigned to monitor whether a signal remains stable for a 
specified period of time. Another checker may be used to verify that a consequent expression holds only if the antecedent expression holds.

The use of assertion checkers, however, leads to a resource overhead and a performance lag based on the number and size of the checkers implemented. This performance drawback has to be considered when implementing ABV.

\subsection{Problem statement}

An FPGA has limited I/O and logic resources that need to be strategically utilized when implementing a design. As the size of an FPGA-based design increases, it reaches a design bottleneck due to an I/O pin or a logic resources constraint. An FPGA-based shifter is an I/O intensive design. Thus, the I/O limitations of an FPGA restrict the design of large FPGA-based shifters.

This thesis explores the use of a novel algorithm to alleviate this bottleneck. The algorithm should be synthesizable into an FPGA-based design. The design should be optimized for an FPGA and scalable to a multiple-FPGA chip setup to improve performance or to implement a larger shifter.

The use of assertion checkers within the shifter design is also explored. The thesis utilizes assertion checkers from the Open Verification Library (OVL). The performance metrics of individual OVL checkers are studied and tabulated in a datasheet for reference and further research.

Furthermore, a novel approach that reduces the overhead caused by assertion

modules in an FPGA-based design is explored. This approach should have the advantages of an assertion based design with performance characteristics comparable to a conventional assertion-free design. 


\subsection{Thesis organization}

Chapter 2 reviews the research previously undertaken in shifter design and assertion based verification. Chapter 3 provides a brief overview of FPGAs and FPGA programming. The characteristics of the FPGA family used in this thesis are described and an overview of the steps for implementing FPGA-based designs is discussed. The various types of hardware modeling flows are then enu merated.

Chapter 4 provides an understanding of the various shifter designs currently used and the design bottlenecks encountered when implementing FPGA-based shifters. Chapter 5 proposes a novel shifter design that alleviates FPGA-based design bottlenecks. The HDL structure and design of the common shifters and the proposed design are elaborated in Chapter 6 and the performance metrics of the implemented HDL designs are tabulated. Chapter 7 discusses the challenges during design verification and explains the use of the assertion-based verification methodology. The OVL assertion checkers that are inserted in the proposed shifter design are described. Each VHDL OVL checker is synthesized individually to obtain a performance datasheet for the checkers. The generated data is used to estimate the performance overhead in an assertion-based shifter design. Chapter 8 proposes and implements a novel method to alleviate the resource overhead and delay due to assertions. The performance of the assertion-based design is compared against the performance metrics estimated in Chapter 7. 


\section{Chapter 2}

\section{Literature survey}

\subsection{Shifter design}

The barrel shifter first proposed by Lim in 1972 [1] uses a single stage to shift the input data. This results in a complicated design that increases power consumption. A logarithmic shifter design alleviates the power dissipation problem [3] and also occupies less chip area [2]. Since the shift operation forms an integral component of any arithmetic logic unit, researchers have explored several variations of the barrel shifter and logarithmic shifter design to suit their application needs.

Tharakan and Kang [4] presented a variation of a 32-bit logarithmic shifter that uses ternary logic. A design that shifts left or right by 1, 3 and 9 bits is proposed as opposed to using conventional binary logic that shifts in logarithmic powers of two. This

combination is used to perform a left or right shift of any magnitude less than 32 . The resulting CMOS logic design demonstrates a $30 \%$ increase in speed but increases the required chip area by $17 \%$.

Yih et al. proposed a variation of the barrel shifter for use in a CORDIC computer [5]. The primary objective of the design is to reduce the chip area. A 2-level barrel shifter is proposed that processes the data in 2 stages connected in series. The proposed design reduces the size of the CORDIC shifter by $68 \%$.

Beerel et al. present a low power asynchronous barrel shifter design for use in multimedia applications [6]. The objective of the design is to reduce its power 
consumption during MPEG video processing. A distribution chart for a sample set of video files showed that $85 \%$ of the codewords were of length 6 bits or less. The barrel shifter was split in two levels to optimize for this codeword length. The shifter utilizes only the first level if the codeword length is 6 bits or less. The two levels are used simultaneously for a larger codeword. The design results in a $61 \%$ reduction in the average energy consumed.

Finally, the design of common shifters is summarized by Pillmeier [7]. The delay and chip area characteristics of the designs are then compared.

\subsection{Assertion-based verification}

Assertion Based Verification (ABV) is used in both software and hardware verification and has thus been promoted by researchers in both fields. The assertion-based methodology for hardware verification was originally developed by Harry Foster [8]. The advantages of $\mathrm{ABV}$ in reducing the design verification time is discussed in [8] [9]. Meyer [10] addresses the use of assertions in Object Oriented Programming (OOP). The paper suggests the use of assertion checks at points where the sub-program is called by the main program. Failure to comply with the assertion returns the control to another subprogram that handles such special cases in an assertion checker format. [11] describes the use of ABV in System on Chips (SoCs). Each SoC component presents unique verification problems. For example, resource allocation requires proper semaphore logic to lock resources and prevent conflict. To detect possible conflict scenarios, the behavioral assumptions are modeled using assertions. Assertions are further used in design interfaces help gather information on the data being transferred. This assists in designing systems linked through an interface. 
[12] provides an empirical analysis of assertion use. It proposed the use of assertions only at the critical points in the design to create a minimal overhead and speed reduction. Riazati et al. have synthesized assertions for a basic CPU design for detection of stuck-at faults and presented the overhead resources needed for their implementation [13]. The effectiveness of an assertion checker is measured through a performance metric that is proportional to the area overhead and fault coverage of the corresponding checker. 


\section{Chapter 3}

\section{Field Programmable Gate Arrays}

\subsection{Introduction}

A field-programmable gate array (FPGA) is a semiconductor device containing programmable logic blocks and programmable interconnects. The logic blocks can be programmed to perform the function of basic logic gates or more complex combinational functions such as decoders or simple mathematical functions.

FPGAs are usually slower than their Application-Specific Integrated Circuit (ASIC) counterparts. They cannot handle as complex a design, and draw more power. However, FPGAs have a shorter time to market and the ability to re-program in the field. This lowers non-recurring engineering costs. As FPGA size, capabilities and speed have increased, it has allowed implementation of larger functions until some FPGAs are now marketed as a full System on Chip (SoC) [16].

\subsection{Applications}

FPGAs are increasingly being used in conventional High Performance Computing applications where computational kernels such as FFT or Convolution are performed on the FPGA instead of a microprocessor. The use of FPGAs for computing tasks where time intensive tasks are offloaded from software to FPGAs is known as reconfigurable computing [26]. The current (2007) generation of FPGAs can implement $~ 100$ single precision floating point units, with each unit computing a result every clock cycle. The 
flexibility of the FPGA allows for even higher performance by trading precision for an increased number of parallel arithmetic units [17].

The adoption of FPGAs in high performance computing is currently limited by the complexity of FPGA design compared to conventional software and the extremely long turn-around times of current design tools. A 4-8 hour wait is necessary after even minor changes to the source code of the FPGA.

\subsection{Manufacturers}

The main manufacturers of general purpose FPGAs are Xilinx [27] and Altera [28]. Other manufacturers for special purpose FPGAs include Lattice Semiconductor [29], Actel [30], and Atmel [31].

Table 3.1: Characteristics of the Spartan-3 Series

\begin{tabular}{|l|l|l|}
\hline Spartan-3 devices & Logic Cells & Usage \\
\hline Spartan-3 & 1700 to 75000 & $\begin{array}{l}\text { For high density and high pin count } \\
\text { applications }\end{array}$ \\
\hline Spartan-3A & 1500 to 25000 & I/O optimized \\
\hline $\begin{array}{l}\text { Spartan- } \\
\text { 3AN }\end{array}$ & 1500 to 25000 & Non volatile memory packaged with a 3A \\
\hline Spartan-3E & 2000 to 33000 & Logic optimized \\
\hline Spartan-3A DSP & $\begin{array}{l}37000 \text { to } \\
54000\end{array}$ & DSP optimized \\
\hline
\end{tabular}

Xilinx is the leading FPGA manufacturer with device families for glue logic (CoolRunner, CoolRunner II), low-cost (Spartan) and high-end (Virtex) applications. The Virtex-II, Virtex-4 and Virtex-5 FPGA families are used to design System-on-Chip (SoC) as they can be embedded with two IBM PowerPC cores [26]. The Spartan series FPGAs are slower than the corresponding Virtex FPGAs and do not support embedded PowerPC cores. However, as a low-cost FPGA family, it offers an economical alternative. 
The current generation of Spartan-3 FPGAs is fabricated using 9onm technology. The FPGA families within the series are grouped according to the general use as shown in Table 3.1.

\subsection{The Spartan-3A series}

The Spartan-3A series offers low logic densities coupled with higher I/O pin count. This configuration is suitable for designing shifters due to similar design requirements. The Spartan-3A family is comprised of the FPGAs detailed in Table 3.2. The research work in this thesis is performed using the Xilinx Spartan-3A family of FPGAs.

Table 3.2: Characteristics of the Spartan-3A family

\begin{tabular}{|l|c|c|c|}
\hline Device & Package & I/O pins & Slices \\
\hline $\mathrm{XC}_{3}$ S50A & FT256 & 144 & 704 \\
\hline $\mathrm{XC} 35200 A$ & FG320 & 248 & 1792 \\
\hline $\mathrm{XC}_{3}$ S400A & FG400 & 311 & 3584 \\
\hline $\mathrm{XC} 3 \mathrm{~S} 700 A$ & FG484 & 372 & 5888 \\
\hline $\mathrm{XC} 3 \mathrm{~S} 1400 A$ & FG676 & 502 & 11264 \\
\hline
\end{tabular}

\subsection{FPGA programming and design approach}

The design of an integrated circuit is typically performed using Electronic Design Automation (EDA) tools. The general flow of an EDA process is shown in Figure 3.1. Initially, the functional objectives of the chip are decided. The architecture of the circuit is implemented at Register Transfer Level (RTL). For ASICs and FPGAs, this is performed using Hardware Description Languages (HDL). The hardware description is then verified for proper behavior and functioning. Any corner cases or behavioral faults are eliminated at this stage through functional verification, assertion based verification, simulation etc. 
The RTL design of the chip is then used to create a floorplan of the design. The input/output (I/O) pins are assigned and objects such as arrays and cores are placed. The logic synthesis process generates a design netlist from the HDL design to describe the connectivity of the design. The netlist is then used to perform a Place-And-Route (PAR). The placement process assigns exact locations for design components. An inferior placement assignment affects the performance of the design and may make it impossible to manufacture the design due to exhaustion of routing resources. The routing process adds the wiring to connect the gates in the netlist. The FPGA is further optimized to meet timing requirements or eliminate any violations such as noise and yield [18] [19].

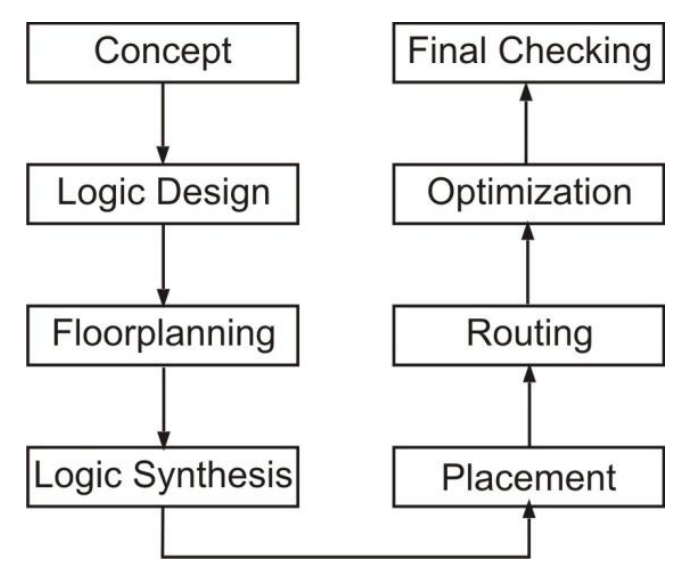

Figure 3.1: EDA Design Flow

FPGA-design tools are of two types: the proprietary software from the FPGA manufacturer or third-party software. The third-party software tends to be expensive while offering better technical support. The FPGA manufacturer's proprietary software is usually available for free to promote the corresponding products. However, the netlists generated from any proprietary software can only be used exclusively with that software. Xilinx offers a proprietary EDA tool named Xilinx ISE, while Altera uses its Quartus II software. The Quartus II software features include implementation, device fitting, and 
JTAG programming solutions. The Xilinx ISE offers HDL synthesis and simulation in addition to the features available in Quartus II.

Since the thesis research is being performed on the Xilinx Spartan-3A family, the proprietary FPGA tools for it are needed. The Xilinx ISE 9.2i Webpack is thus used for performing the synthesis and implementation of the HDL designs in the remainder of the thesis.

\subsection{HDL modeling}

The three distinct types of modeling used in VHDL are structural modeling, dataflow modeling and behavioral modeling [15]. Structural modeling describes the design as a set of interconnected components without a description of the component behavior. This model thus represents the hierarchy of the design accurately but lacks the description of the design behavior. A structural model is implemented through component instantiation within the parent entity. The component instances are connected to parent components through their ports to form an interconnected design.

The dataflow modeling system utilizes concurrent signal assignment statements to describe the flow of the data. The design structure is not explicitly stated in the dataflow model, but can be deduced from the flow of the data through the model.

The behavioral or functional modeling process represents the system as set of sequential functions. This modeling system does not describe the design hie rarchy of the system in any way. The behavioral description simply gives the relationship between the input and output using functions without any in dication of the design structure.

A typical hardware design utilizes two or more types of modeling descriptions. For example, the structural model may be used to describe the hierarchy of the system 
and the behavior of each sub-system is defined using either dataflow or behavioral modeling. 


\section{Chapter 4}

\section{Shifter Design and FPGA-based Shifter Limitations}

\subsection{Common shifter designs}

A shift register requires as many clock cycles as the magnitude of the shift to be performed. In comparison, a barrel switch [1] operates by shifting the data completely in a single clock cycle. This characteristic of the barrel shifter results in high speed performance required by high-speed processors and graphic and video processing units. The implementation of a barrel shifter in these applications considerably improves the speed of the device.

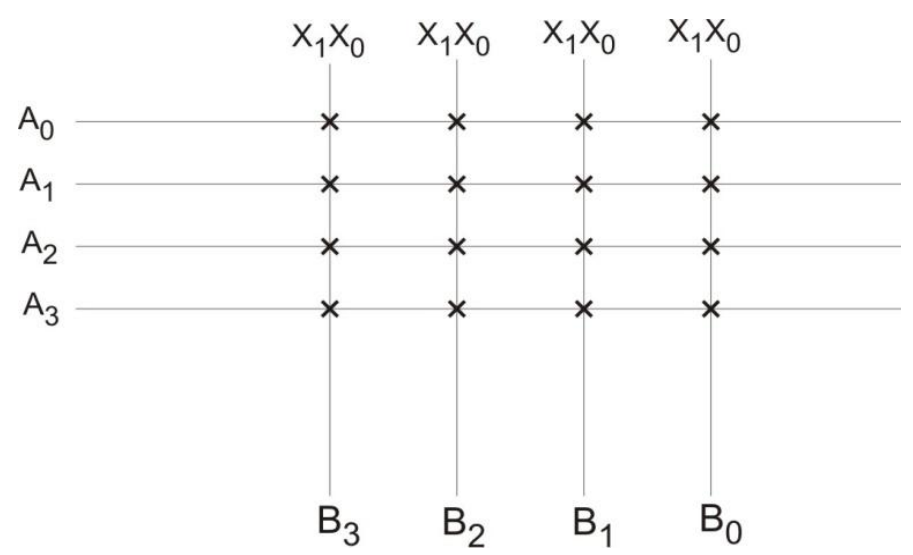

Figure 4.1: Design of a 4-bit barrel switch

A basic barrel shifter consists of logic switches arranged as shown in Figure 4.1. The input $A$ to the barrel switch is transmitted to the output through the switches. The switches are controlled using the shift control $X$ that determine the path of the data 
through the barrel switch. The state of the switches determines the flow of data through the design to generate the output $B$.

The barrel shifter was first introduced in modern devices in the Intel 80386 processor in 1986. It is implemented in modern devices through multiplexers as illustrated for a 4-bit barrel shifter in Figure 4.2. An n-bit barrel switch utilizes $n$-bit multiplexers.
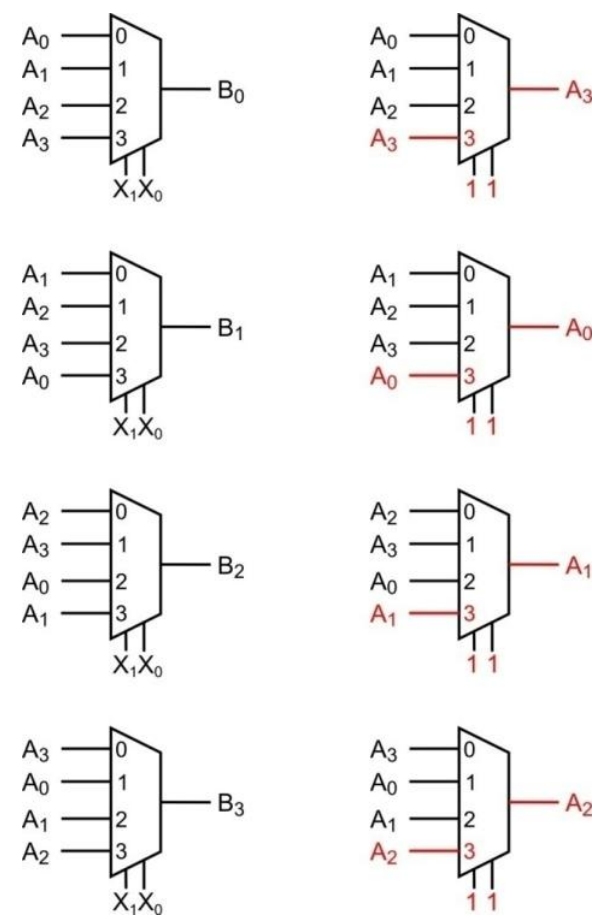

Figure 4.2: Design and working of a 4-bit barrel shifter

Each multiplexer has the same inputs with the arrangement of the input data varying by one position for every multiplexer in the array. The shift control determines the input bit that appears at the output of each multiplexer. This is illustrated in Figure 4.2 where the 4-bit input data is rotated by 3 bits. Each multiplexer has the same input data $A$ and shift control $X$. However, the input data to each multiplexer is incrementally 
rotated by a single bit. The output $B$ of the barrel shifter is thus rotated by the desired amount.

A typical barrel shifter thus has an $n$-bit data input, an $n$-bit data output, and a $\log _{2} n$-bit shift control to determine the magnitude of the shift.

The design of a barrel shifter results in high speed performance but also presents numerous problems. The number of in put lines to the barrel shifter design is $n^{2}$. As the design size increases, the complexity and chip area of the design increases exponentially. The large number of inputs also causes a loading effect at the input source. These effects make the design of large barrel shifters infeasible in real-time applications.

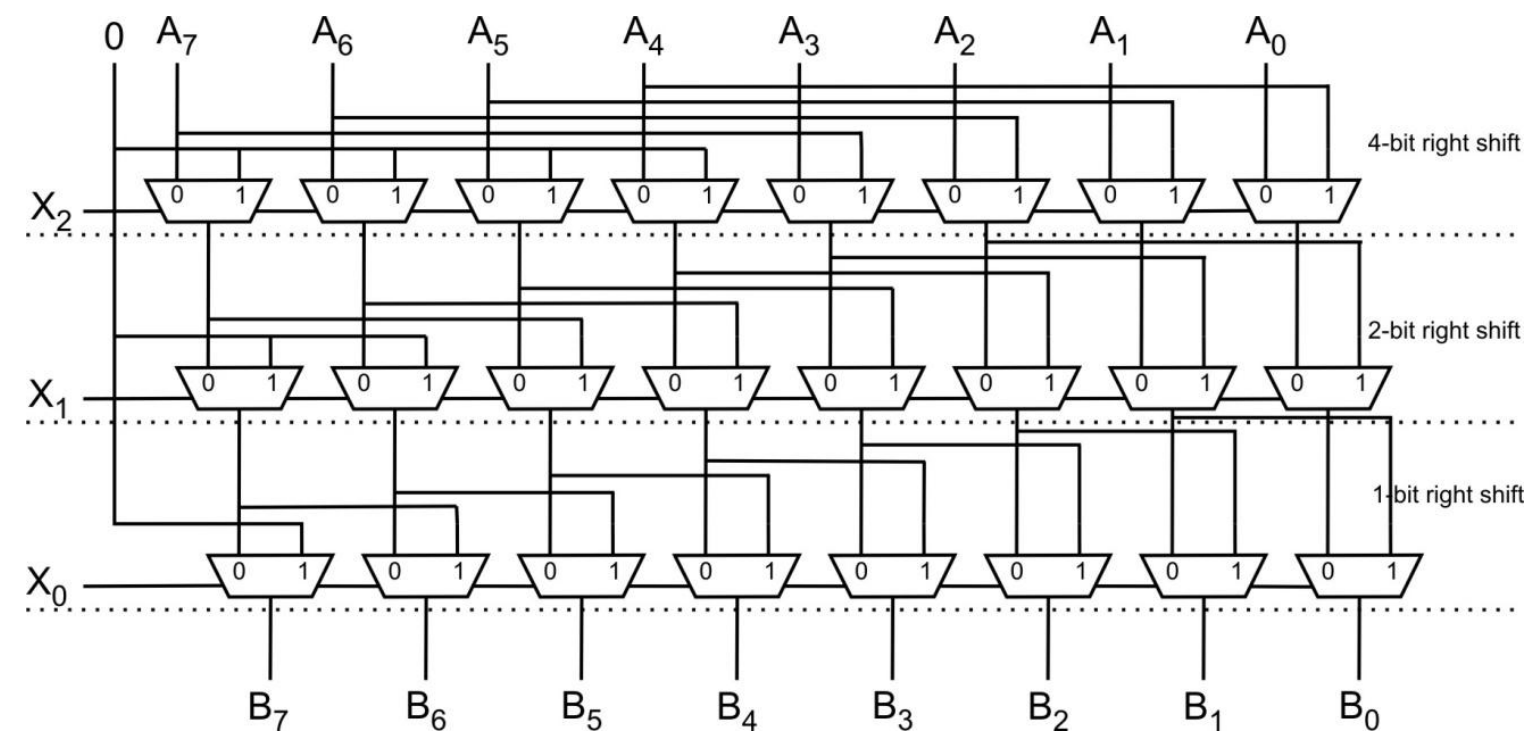

Figure 4.3: Design of an 8-bit logarithmic shifter

The drawbacks of the barrel shifter design are addressed in the logarithmic shifter design. Unlike a barrel shifter, the log shifter shifts the data in stages. Each stage shifts its input by an integer power of two. Thus, an 8-bit log shifter is composed of 3 stages as shown in Figure 4.3. The stages shift the data by 1, 2 and 4 bits using 2-bit multiplexers arrayed in the stages. 
The log shifter design results in reduced power consumption [3] and utilizes less chip area [2] compared to a barrel shifter. However, the increased number of stages leads to a slight reduction in the speed of the log shifter compared to a barrel shifter [2].

The log shifter can be modified to include a rotate functionality in the log shifter. This is performed by connecting $A_{0}$ as input to the multiplexer with $A_{7}$ as input. The rotate and shift functionality can thus be combined together as shown in Figure 4.4.

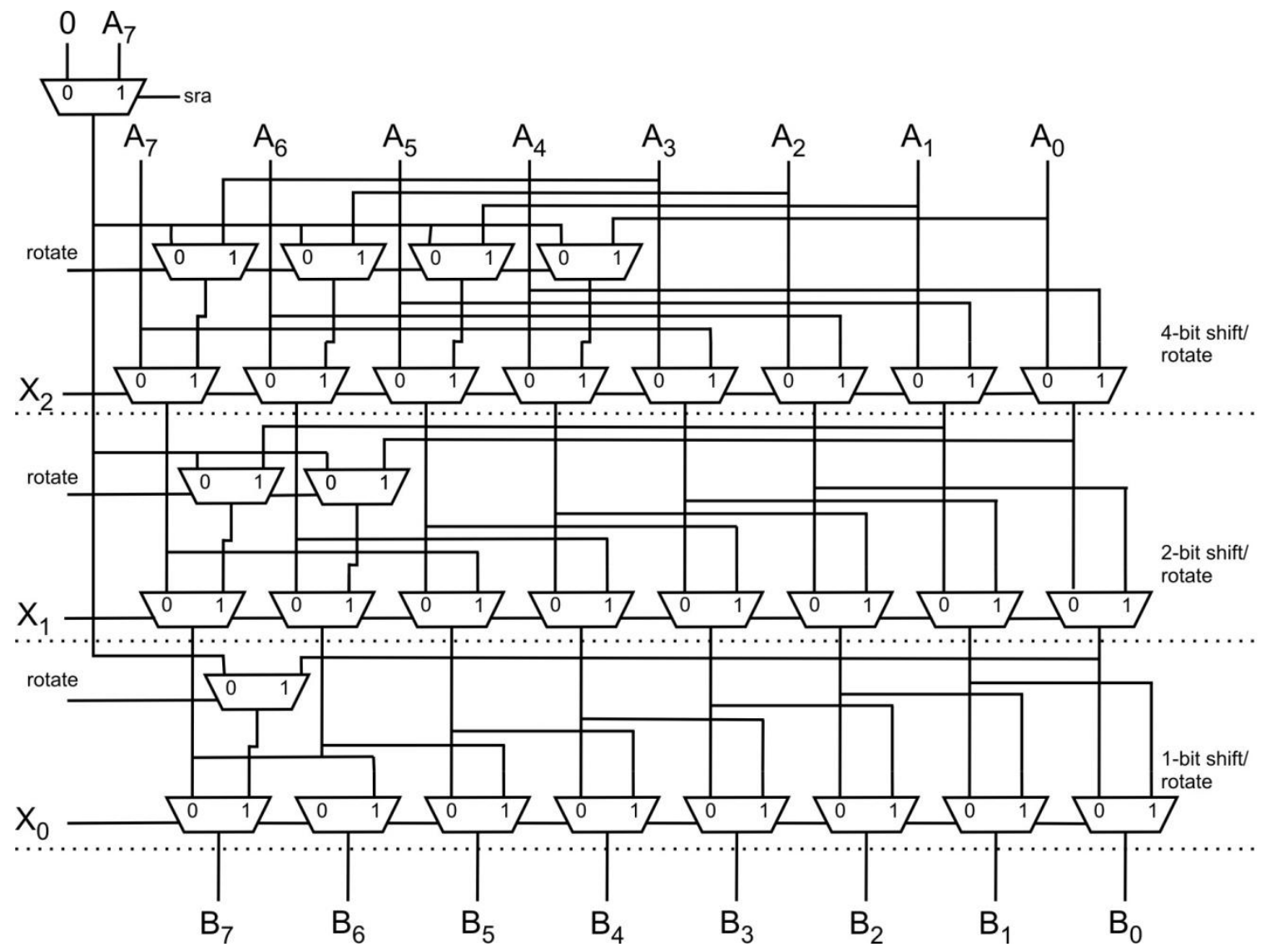

Figure 4.4: Design of an 8-bit logarithmic shifter/rotator

A single multiplexer selects between 'o' when logical right shifting, or $A_{n-1}$ for arithmetic right shifting. In the stage controlled by $\mathrm{X}_{\mathrm{k}}, 2^{\mathrm{k}}$ multiplexers select between ' $\mathrm{O}$ ' (or $A_{n-1}$ ) for shifting and the $2^{k}$ lower bits of the data for rotating [2]. 


\subsection{FPGA-based shifter limitations}

An $n$-bit log shifter/rotator design described in Section 4.1.2 utilizes an $n$-bit input, a $\log _{2} n$-bit shift control, a 2-bit opcode and an $n$-bit output. The opcode determines the type of shift/rotate to be performed. The total number of I/O pins $r$ utilized by the shifter when implemented on an FPGA is,

$$
r=2 n+\log _{2} n+2
$$

Since a shifter design is I/O intensive, the number of FPGA I/O pins available is the common bottleneck for an FPGA-based shifter design. The largest FPGA in the Spartan-3A family used in this thesis is the $\mathrm{XC}_{3} \mathrm{~S} 1400 \mathrm{~A}$ with $502 \mathrm{I} / \mathrm{O}$ pins as described in Section 3.4. Thus, the largest shifter that the Spartan-3A family can implement has a 128-bit input. Using a conventional design, a shifter of larger size cannot be implemented on the Spartan-3A FPGAs. To overcome this bottleneck, a novel algorithm is proposed for implementing FPGA-based shifters. 


\section{Chapter 5}

\section{Proposed FPGA-based shifter design}

\subsection{Proposed design of an embedded auxiliary shifter}

In the proposed design, an auxiliary shifter of size $s$ and additional logic are embedded in the FPGA. It is used to process and shift the $n$-bit input. The implementation of the embedded auxiliary shifter is a hybrid design using combinational and sequential logic.

The input data $A$ to be shifted is comprised of $n$ bits, with the $i^{\text {th }}$ bit denoted by $\mathrm{A}_{\mathrm{i}}$, $0 \leq i \leq n-1$. $A$ is partitioned into $\frac{n}{s}$ data blocks with each block denoted by $A^{p}, 0 \leq p \leq$ $\frac{n}{s}-1 . p$ denotes the initial index of the block. Each block consists of $s$ bits with the $j^{\text {th }}$ bit denoted by $A_{j}^{p}, 0 \leq j \leq s-1 . A$ is partitioned such that,

$$
A_{j}^{p}=A_{p+j \times \frac{n}{s}}
$$

The proposed approach partitions the input based on the block number $p$ and the number of data blocks $\frac{n}{s}$. $p$ determines whether a block is comprised of either evenreferenced bits or odd-referenced bits of the input. Every block thus created is processed independently. This is in contrast to using a simple linear approach where,

$$
A_{j}^{p}=A_{p \times s+j}
$$

This would cause the number of input bits needed in a block to be dependent on the magnitude of the operation to be performed. 


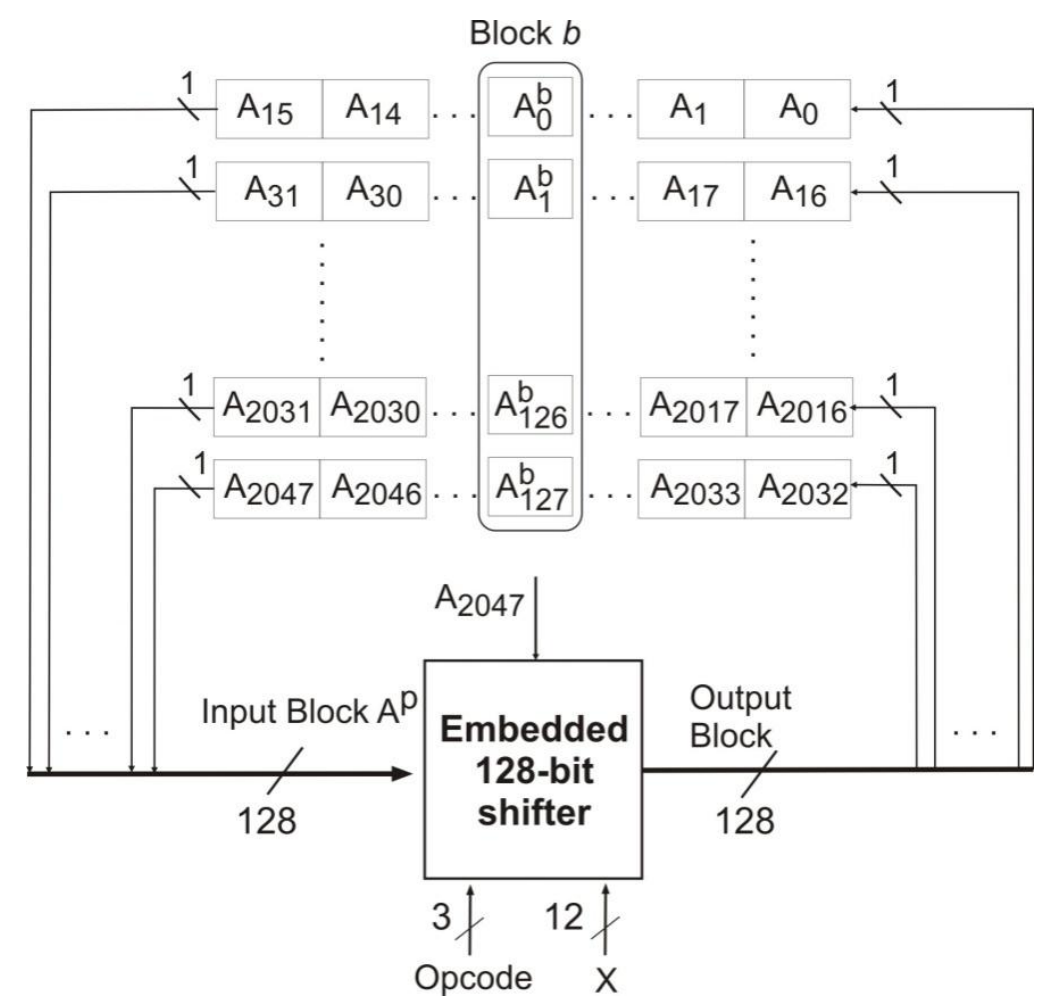

Figure 5.1: Design of a 2048-bit shifter using 128-bit embedded logic

\subsection{Sequential-logic level processing}

Figure 5.1 shows the overview of the proposed design for a 2048-bit shifter implemented using a 128-bit embedded auxiliary shifter and a 2048-bit shift register that stores the data. The blocks are processed in decreasing order of the value of the corresponding $p$. The register first inputs the block $A_{\frac{n}{s}-1}$ (in this case, $A_{15}$ ) to the FPGA where it is processed by the embedded auxiliary shifter.

This generates an updated index $q$ for that specific block. It is stored along with the processed block within the FPGA and the value of $p$ is decremented by 1 . The next block is then input. This sequence of operations is iteratively carried out until block $A_{\mathrm{o}}$ is processed. The synthesis of the desired shifted output now follows. The block with $q=\frac{n}{s}-1$ is output followed by the remaining blocks in decreasing order of the value of 
the corresponding $q$. If the maximum delay to process and store a block is $d$, then the total processing time $t$ required for the $n$-bit in put is,

$$
t=\frac{n}{s} \times d
$$

\subsection{Embedded auxiliary shifter and shift-control partitioning}

The inputs to the embedded auxiliary shifter are the $s$-bit data block, a 3-bit opcode $O$, an $m$-bit primary shift-control $X\left(m=\log _{2} n\right)$ and $A_{\mathrm{n}-1}$, the most-significant bit of the input $A$. While $X$ determines the magnitude of the shift, $O$ specifies the direction (left/right) and the type of shift operation (rotate/arithmetic shift/logical shift). In addition to these inputs, the shifter uses the initial index $p$ and generates an updated index $q$ as described

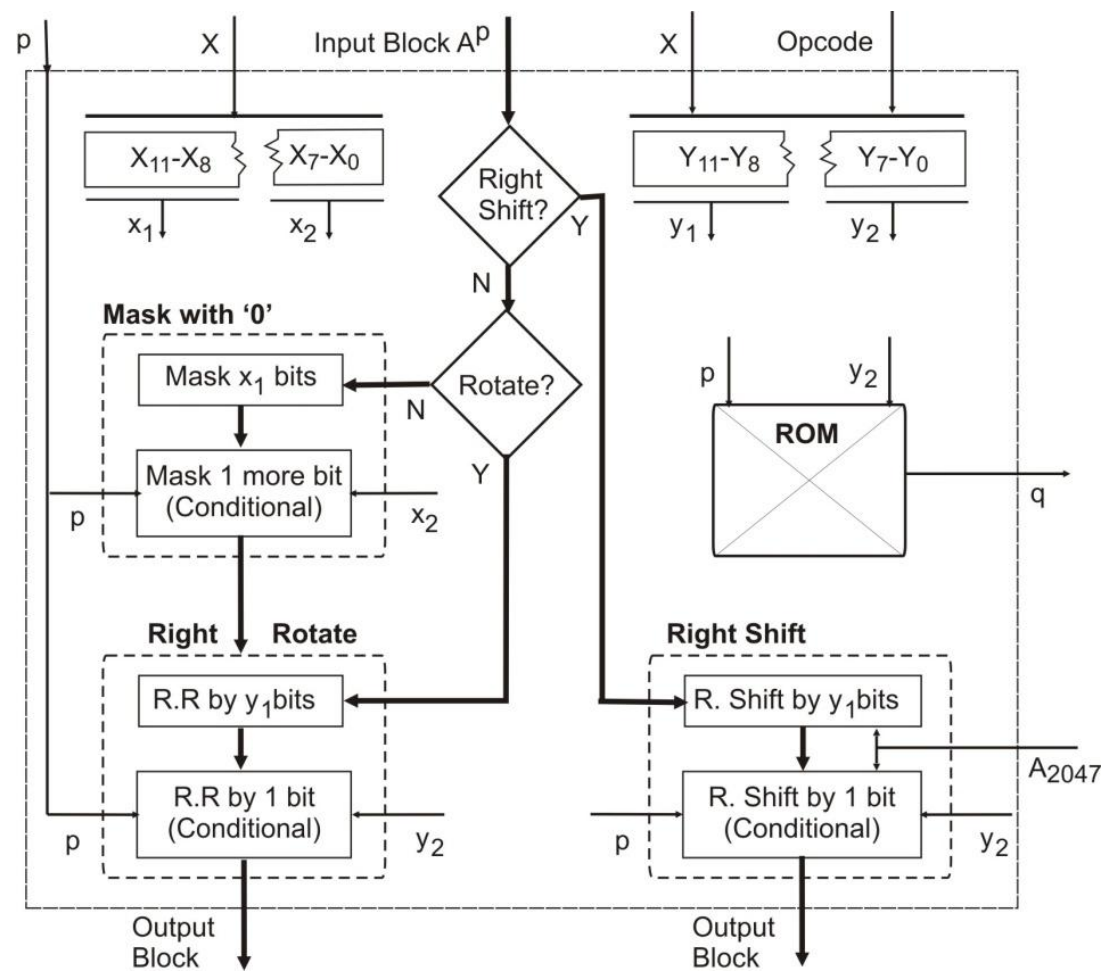

Figure 5.2: Control flow of the combinational logic 
in Section 5.2. A secondary shift control $Y$ maps to $X$ if the direction of shift is right, or maps to 2's complement of $X$ if the direction of shift is left.

The primary operation performed by the shifter is right shift and right rotate. In a right arithmetic shift, the block is appended with $A_{n-1}$ as its most-significant bit and the arithmetic shift is performed. In case of a left shift, the $X$ most significant bits are masked with ' 0 '. The data is then rotated right by $Y$. A left rotate is performed by rotating right by $Y$.

The embedded auxiliary shifter thus uses the shift-control $X$ (or $Y$ ) to perform a mask (or shift/rotate). However, using an $m$-bit shift-control could lead to a mask (or shift/rotate) greater than the size of the $s$-bit data block. To correct this, the shift-control is partitioned into two independent bitstrings which are then used independently. The first bit-string is comprised of the $\log _{2} s$ most-significant bits of $X$ (or $Y$ ). Its integer value is denoted by $x_{1}$ (or $y_{1}$ ). The second bit-string is comprised of the remaining $m-\log _{2} s$ least-significant bits. Its integer value is denoted by $x_{2}$ (or $\left.y_{2}\right)$.

\subsection{Combinational-logic level processing}

Figure 5.2 shows the flow of control through the combinational-logic level of the embedded auxiliary shifter. The first set of operations carried out on a block is to perform bit-masking with zeroes. It is performed only in case of a left shift. As a first step, the $x_{1}$ most-significant bits of the block are masked. The second step involves conditional masking of one additional bit. This is performed only if,

$$
p \geq \frac{n}{s}-x_{2}
$$

The second set of operations involves either right shifting or right rotating. It is performed on all blocks irrespective of the type of shift. If the operation is right 
arithmetic shift or right logical shift, then the corresponding operation is performed by an amount equal to $y_{1}$. For every other operation, the block is rotated right by $y_{1}$. The right arithmetic shift for a block is always performed after appending $A_{\mathrm{n}-1}$ as its mostsignificant bit.

The second step involves conditional right shift (or right rotate). The data block is right arithmetic/logical shifted (or right rotated) by another bit if $p<y 2$.

These two sets of operations when performed result in an appropriately shifted/rotated block.

\subsection{Index updating}

Before processing each block, $q$ is initialized to the same value as the block's corresponding $p$. When performing a conditional right shift/rotate, it's value is decremented by $y_{2}$. However, it is always in the range,

$$
0 \leq q \leq \frac{n}{s}-1
$$

If it reaches ' 0 ' during the operation, the count resumes from the highest value, $\frac{n}{s}-1$.

\subsection{Design optimization}

To minimize the processing time and the logic resources used, the following optimizations are performed:

1. The first step of both sets of operations described in Section 5.4 masks (or right shifts/rotates) the data block by $x_{1}$ (or $y_{1}$ ) in a single stage. This is similar to a barrel shifter design. These steps may be performed in a series of smaller stages so that the total mask (or right shift/rotate) performed is equal to $x_{1}$ (or $y_{1}$ ). This 
reduces the complexity of the design, and consequently the FPGA slices used. The variation used in this research is similar to a logarithmic shifter where a series of stages shift data in integer powers of two. To further reduce the delay in a logarithmic design, the stages are arranged in decreasing order based on the magnitude of shift performed in each stage. Experimentally, this arrangement is generally faster with up to $37.8 \%$ lower processing time and $5.3 \%$ fewer slice usage compared to a design with progressively increasing order of shifts in each stage.

2. The updated index $q$ changes value when a block is processed at the combinational-logic level. To attain the necessary speed, an embedded ROM is used to determine the updated value of $q$.

3. The total FPGA pins $r$ used by an embedded auxiliary shifter of size $s$ is,

$$
r=2 s+\log _{2} s+4
$$

When using an FPGA with $f$ I/O pins, $s$ is optimized to maximize the amount of data transferred and thus minimize the time taken to process the entire data. The ratio $\frac{r}{f}$ denotes the percentage of I/O pin utilization.

4. The performance in speed of the proposed shifter design is improved when the size of the embedded logic is increased.

5. The number of I/O pins and slices utilized by the proposed shifter design is reduced when the size of the embedded logic is decreased.

\subsection{Extension to multi-chip FPGA design}

The proposed design alleviates the I/O resource bottleneck for an FPGA shifter design. However, as the ratio $n: s$ increases, the total processing time $t$ also increases and may exceed the design constraints. To reduce the delay, a multi-chip FPGA can be used where each chip processes a consecutive set of blocks. This allows multiple blocks to be 
processed simultaneously. When all blocks have been shifted, the desired output is synthesized in the same manner as described for a single FPGA design. 


\section{Chapter 6}

\section{Implementation of Shifter Designs in HDL}

The performance of the proposed design is quantitatively compared against the conventional shifter designs: the barrel shifter, the logarithmic shifter and the shift register. This is performed by implementing the VHDL description of the shifters on an FPGA. The I/O and logic resources used and the performance lag of the four designs are then used to compare the performance. The following section describes the HDL structure and behavior of the shifter designs.

\subsection{Barrel shifter}

The barrel shifter performs the shift using a single stage. This behavior is modeled through functional modeling in VHDL as illustrated in Figure 6.1.

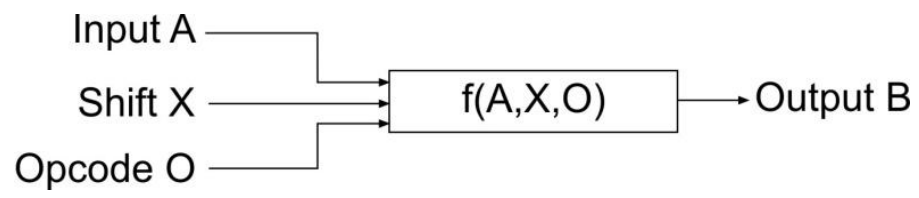

Figure 6.1: HDL design of a barrel shifter

A single function calculates the output based on the input data, the shift magnitude and the opcode. VHDL supports logical operations such as arithmetic shift (sra, sla), logical shift ( $s r l, s l l)$ and rotate (ror, rol) through specific operators. These are used to create a simple relation between the input and output of the barrel shifter. The resulting function calculates the output through a single concurrent signal assignment statement. 


\subsection{Logarithmic shifter}

The HDL description of a log shifter is designed in stages resembling its design layout shown in Figure 6.2. The design uses a mixed modeling system. It utilizes a dataflow modeling system with an underlying functional model. The functional layer models each stage of the log shifter while the dataflow layer simulates the arrangement of the stages to perform the shift/rotate. A concurrent signal assignment statement performs the function of the corresponding stage. These statements are connected in series to form the shifter. The stages are arranged in increasing order based on the magnitude of shift performed in each stage.

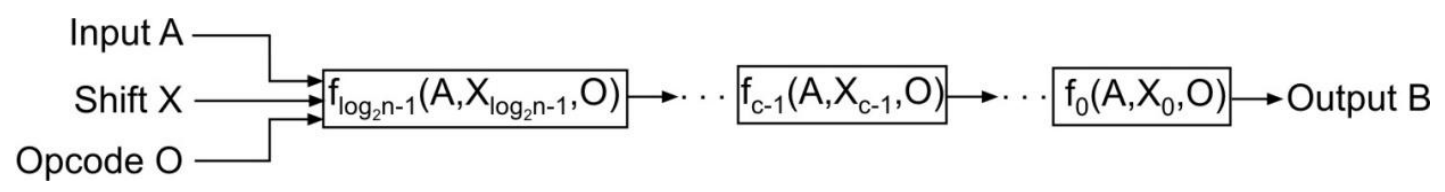

Figure 6.2: HDL Design of a logarithmic shifter

\subsection{Shift register}

A sequential-logic based shift register utilizes a clock to begin a shift cycle at the rising edge of the clock. With each clock cycle, a shift/rotate by one bit is performed based on the opcode and shift control.

The HDL design of the shift register utilizes functional modeling. The shift

process is described within a process statement with the clock signal included in the sensitivity list. A case statement performs the shift/rotate operation based on the opcode value at each rising edge of the clock signal. 


\subsection{Proposed auxiliary shifter design}

The proposed design utilizes sequential logic and combinational logic as described in Chapter 5. The combinational logic is encapsulated within the sequential logic. This hierarchy is preserved when implementing the design in VHDL. Figure 6.3 illustrates the HDL structural description of the proposed shifter. The sequential logic of the shifter is

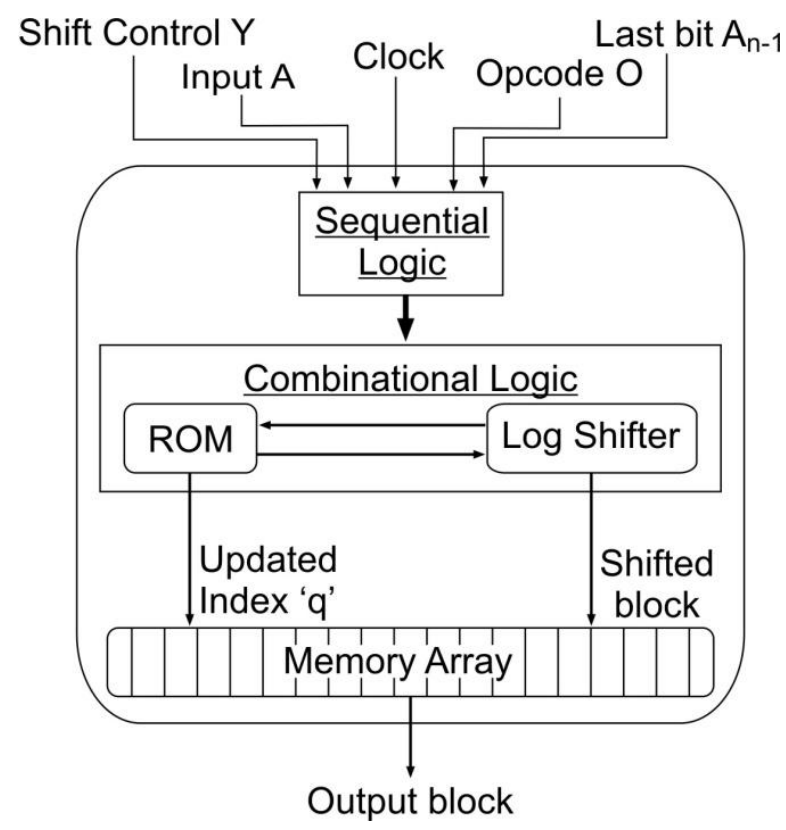

Figure 6.3: HDL hierarchy of the auxiliary shifter

described by an entity sequential_logic. This entity controls the data processing performed by the combinational logic components: the built-in ROM and the log shifter. These two combinational logic components are bound by a single entity named combinational_logic.

\subsubsection{Sequential_logic}

This is the top-level entity defined for the shifter. Its inputs are the input block data, shift control, opcode, lastbit, a clock signal, a reset pin and the output block. This design utilizes a clock signal to input a block and store the processed output of 
combinational_logic. The reset bit is set to active high to reset the shift process. When set to active low again, the shift process starts with the first block input to combinational_logic. The processed output is stored and the next block is then input at the rising edge of the next clock cycle. The remaining process proceeds as described in Section 5.2.

\subsubsection{Combinational_logic}

The combinational_logic entity receives the in put from sequential_logic, shifts the data by the requisite amount and outputs it to the memory array.

The log shifter within combinational_logic has an identical HDL structure and the same functionality as the log shifter in Section 6.2. However, its stages are arranged in the descending order of the magnitude of shift performed in each stage. This arrangement is faster compared to an increasing order of stages. The ROM component of combinational_logic is comprised of the following entities:

1. Next_block_index: Returns an integer value to combinational_logic. It calculates the current index of the next block that is to be input to the shifter.

2. Mask_most_sig: When performing a left shift, the most significant bits of the input are masked first. The magnitude of this mask is the integer value of the $\mathrm{y}_{1}$ most significant bits of amount. This value is calculated by the mask_most_sig entity and returned to combinational_logic.

3. Least_sig_value: This entity calculates the integer value of the $\mathrm{y}_{2}$ least significant bits and returns it to combinational_logic during a conditional mask operation.

4. Shift_rotate_value: This entity calculates the integer value of $y_{c}^{2}$, and returns it to combinational_logic during a conditional shift/rotate operation.

5. Updated_index_u: This entity calculates this updated index $u$ of the block. 


\subsection{Experimental results}

The Xilinx Spartan-3A family of FPGAs [8] provides a cost-effective FPGA design solution. The research in this section uses two types of FPGAs from the Spartan-3A family. The $\mathrm{XC}_{3} \mathrm{~S} 2 \mathrm{OoA}$ has $248 \mathrm{I} / \mathrm{O}$ pins and 1792 logic slices and the $\mathrm{XC}_{3} \mathrm{~S} 400 \mathrm{~A}$ has 311 I/O pins and 3584 logic slices. To evaluate the performance of the proposed shifter design, its delay, I/O, and logic resources utilization are compared with a shift register, a barrel shifter, and a logarithmic shifter design.

Table 6.1: Comparing performance metrics of the proposed FPGA approach with existing shifter designs

\begin{tabular}{|c|c|c|c|c|c|}
\hline $\begin{array}{l}\text { Shifter } \\
\text { Design }\end{array}$ & $\begin{array}{l}\text { Shifter } \\
\text { Size } \\
\text { (bits) }\end{array}$ & $\begin{array}{l}\text { FPGA } \\
\text { Type }\end{array}$ & $\begin{array}{l}\text { I/O } \\
\text { utilization } \\
r / f \%\end{array}$ & $\begin{array}{l}\text { Total } \\
\text { slices } \\
\text { used } \\
\%\end{array}$ & $\begin{array}{l}\text { Total } \\
\text { processing } \\
\text { delay } t \\
\text { (ns) }\end{array}$ \\
\hline Shift Register & 128 & $400 \mathrm{~A}$ & $86 \%$ & $2 \%$ & 1083.9 \\
\hline Barrel shifter & 128 & $400 A$ & $86 \%$ & $69 \%$ & 10.63 \\
\hline Log shifter & 128 & $400 A$ & $86 \%$ & $45 \%$ & 9.94 \\
\hline \multirow{4}{*}{$\begin{array}{l}\text { Proposed } \\
\text { design: 64-bit } \\
\text { auxiliary } \\
\text { shifter }\end{array}$} & 128 & $200 A$ & $56 \%$ & $44 \%$ & 11.52 \\
\hline & 256 & $200 A$ & $56 \%$ & $44 \%$ & 23.36 \\
\hline & 512 & $200 A$ & $57 \%$ & $45 \%$ & 53.98 \\
\hline & 1024 & $200 A$ & $57 \%$ & $49 \%$ & 98.88 \\
\hline \multirow{4}{*}{$\begin{array}{l}\text { Proposed } \\
\text { design: 128-bit } \\
\text { auxiliary } \\
\text { shifter }\end{array}$} & 256 & $400 \mathrm{~A}$ & $86 \%$ & $50 \%$ & 15.17 \\
\hline & 512 & $400 A$ & $86 \%$ & $49 \%$ & 34.53 \\
\hline & 1024 & $400 \mathrm{~A}$ & $87 \%$ & $50 \%$ & 61.52 \\
\hline & 2048 & $400 \mathrm{~A}$ & $87 \%$ & $51 \%$ & 138.69 \\
\hline
\end{tabular}

Table 6.1 summarizes the experimental results. Using the 400A FPGA, the largest conventional shifter that can be designed is of 128 bits. However, with the proposed design, a shifter of 2048 bits is easily implemented using the same FPGA with an increase of only $1 \%$ in the $\mathrm{I} / \mathrm{O}$ pin utilization. The design complexity can be inferred through the FPGA slices utilized.

The regular shift register uses only $2 \%$ of the FPGA slices available and the barrel shifter uses the most slices at $69 \%$. In contrast, the proposed shifter design uses a 
maximum of $51 \%$ slices when implementing a 2048-bit shifter. Table 6.1 also compares the performance of various shifter designs and the effect of varying the size of the embedded logic. Among the 128-bit shifters, the shift register is the slowest with a delay of $1083.9 \mathrm{~ns}$ due to sequential design, while the log shifter is the fastest with a delay of $9.94 \mathrm{~ns}$. The delay for the proposed design is a modest $11.52 \mathrm{~ns}$ when using a 64-bit embedded shifter.

When the size of the embedded logic is increased from 64-bits to 128-bits, the performance of the proposed design improves by at least $35 \%$ for 256,512 and 1024-bit shifter designs. The FPGA slices used, however, increase by an average of $115 \%$. The results show that with the proposed design approach, larger shifters can be implemented without any I/O bottleneck. The design is optimized for improved performance and minimal area overhead. 


\section{Chapter 7}

\section{The VHDL Open Verification Library}

Any hardware design implemented in HDL needs to be verified for the desired functional behavior before the implementation process proceeds. The drawbacks of an improper verification process include behavioral faults in the design and in extreme cases, design failure. The verification process may comprise simulation, formal verification, assertion based verification etc., or a combination of various methodologies. The ease of verification of any design is primarily dependent on two factors: controllability and observability.

\subsection{Controllability}

Controllability refers to the ability to influence an embedded finite state-machine, structure, or specific line of code within the design by stimulating various input ports. In theory, a simulation testbench has high controllability of the design model's input ports during verification. However, it can have low controllability of an internal structure within the model.

\subsection{Observability}

Observability refers to the ability to observe the effects of a specific internal finite statemachine, structure, or stimulated line of code. Thus, a testbench generally has limited observability if it only observes the external ports of the design model. This is because the internal signals and structures are invisible to the testbench. To identify a design error using the testbench approach, the following conditions must hold: 
1. The testbench must generate proper input stimulus to activate a bug.

2. The testbench must generate proper input stimulus to propagate all effects resulting from the bug to an output port [20].

\subsection{Challenges during verification}

The design complexity of current ASICs and System-on-Chips has increased at a rapid rate leading to a significant increase in the time taken to verify the design. While synthesis tools have increased the productivity of design engineers, the same breakthrough has not occurred in the verification field. Due to this, verification of current designs poses a significant challenge to design engineers. In today's designs, verification makes up to $70 \%$ of the design development time [14].

Consider a 32-bit comparator. The number of input data combinations for the comparator is $2^{64}$. To test for a worst-case single-bit bug, every input combination needs to be used as a test vector. Using a one-million cycle-per-second simulator would take approximately 600,000 years to check for all possible single-bug errors [21]. A complex design would take an even more impractical amount of time for complete verification.

The verification problem is rooted in the traditional approach to verification: A set of input test vectors is used in a design simulation to monitor the actual output against the desired output. The test bench is designed to take account of all corner cases that may possibly occur in the design operation. Thus, the test bench needs to utilize a very large number of test vectors to verify any design. For large designs, the number of test vectors needed becomes impractically large. A simple bug may require an enormous number of test vectors to stimulate it. This results in low controllability.

Another problem facing the traditional verification approach is when internal errors do not propagate to the output making the errors unobservable. Even if the error 
is deduced, it takes a large amount of time to generate and use the test vectors necessary to pinpoint the cause and location of the error.

Finally, when using formal verification processes, there are points within a design that cannot be stimulated using test vectors. These verification 'hotspots' thus cannot be detected with test vectors. Failure to recognize them may render the design in capable of performing its intended task [22].

\subsection{Assertion-based verification}

Assertion-based verification $(\mathrm{ABV})$ is used to reduce the time taken for design verification. It utilizes assertion modules that are inserted at strategic points in the design to maximize their effectiveness. Assertions can be used either as targets for formal proof or as constraints. When used as constraints, assertions define the legal input behavior for the design under verification. Formal-verification tools then exhaust all possible inputs that satisfy the constraints during the process, thereby verifying assertions.

$\mathrm{ABV}$ thus increases the observability of the design by allowing the verification engineer to monitor the internal signal propagation of the design. Using ABV, the internal components of the design can be verified individually. This leads to increased design observability and an enormous reduction in the number of test vectors needed to verify the complete design. Thus, ABV has several advantages over other verification methodologies including,

1. Increasing observability of bugs.

2. Increasing controllability.

3. Finding bugs earlier in the design cycle. 
4. Uncovering bugs that would have otherwise remained undetected.

5. Preventing wasted simulation cycles.

6. Improving verification productivity.

7. Facilitating the integration of work from multiple designers.

8. Supporting design reuse and third part Intellectual Property.

Since the assertions are included as the design is created, formal verification can be started at an early stage without waiting for the completion of the design. Assertions can also be used at the design boundaries to provide checks for interface behavior.

\subsection{Assertion checker standards}

VHDL and Verilog allow the construction of assertion modules through the use of the assertion statement. This statement can be used to check boolean functions in basic monitoring scenarios. However, when assertions are required to monitor more complex tasks such as signal processing, data transfer, common modes of usage or operation and reporting, the built-in assertion statement falls short. A standardized set of assertion modules addresses this problem. Furthermore, a standard set of rules increases the portability of the design. This allows ease of use and transparency in the design. Assertion modules can then be structured so that they do not interfere with the functioning of the design or the synthesis results.

A standardized set of assertions also reduces the need for creation of assertion modules each time a new design is created. A library of such modules allows the user to simply instantiate a module where needed. Additional mechanisms such as controlling the error reporting parameters, the number of errors before failure, or 
enabling/disabling a particular type of assertion module can all be implemented using this standardized set. Thus, using a standardized set of assertions has a noticeable impact on the design creation time. Users can further have confidence that once these assertions are inserted into a design, they can be used by many tools and are portable for design re-use [22].

\subsection{Current assertion standards}

The three standards relevant to $\mathrm{ABV}$ are managed by Accellera [23], the organization responsible for driving these standards. They are,

1. The Open Verification Library (OVL)

2. Property Specification Language (PSL)

3. System Verilog Assertions (SVA)

\subsubsection{Open Verification Library (OVL)}

OVL is the only existing assertion-specification standard in Accellera that currently works with any IEEE-1364 (Verilog) and IEEE-1076 (VHDL) compliant simulator. It also works with a growing number of formal-verification tools.

The OVL is a freely downloadable open source library available in two versions. One version contains Verilog modules, and the other version contains VHDL modules. These modules are used to specify properties of an HDL design to be verified, either in simulation or using formal or semi-formal methods. The modules are instantiated as assertion monitors that will flag violations of the specified property. These modules

provide a standard interface for multiple design verification tools, thus enabling a seamless flow. The OVL defines 31 checkers listed in Table 7.1 [24]. 
Table 7.1: Currently available OVL Checkers

\begin{tabular}{|l|l|l|}
\hline assert_always & assert_implication & assert_quiescent_state \\
\hline assert_always_on_edge & assert_increment & assert_range \\
\hline assert_cycle_sequence & assert_never & assert_time \\
\hline assert_decrement & assert_next & assert_transition \\
\hline asset_delta & assert_underflow & assert_unchange \\
\hline asset_ever_parity & assert_odd_parity & assert_width \\
\hline assert_fifo_index & assert_one_cold & assert_win_change \\
\hline assert_frame & assert_one_hot & assert_win_unchange \\
\hline assert_handshake & assert_proposition & assert_window \\
\hline assert_no_overflow & assert_no_underflow & assert_zero_one_hot \\
\hline assert_no_transition & \multicolumn{2}{|l}{} \\
\hline
\end{tabular}

Every assertion library definition contains a severity_level, options, and a message. As an example, the assert_always assertion continuously monitors the test_expr at every positive edge of the triggering event or clock clk. It contends that the specified test_expr will always evaluate TRUE. If test_expr evaluates to FALSE, the assertion will fire (that is, an error condition has been detected in the code).

The OVL checkers available in the OVL VHDL library and the procedure for the checker configu ration are listed in Appendix A.

\subsubsection{X/Z Check in OVL checkers}

Assertion checkers can produce an indeterminate result if a checker port value contains an X (unknown) or Z (high-impedance) bit when the checker samples the port. To assure determinate results, assertion checkers have special assertions for X/Z checks. 
The ovl_never_unknown and ovl_never_unknown_async assertion checker types are specifically designed to verify that their associated expressions have known and driven values. Thus, they perform an explicit X/Z check.

All other assertion checker types have implicit $\mathrm{X} / \mathrm{Z}$ checks. These are assertions that ensure that specific checker ports have known and driven values.

\subsection{Performance characteristics of the VHDL OVL checkers}

Each OVL checker utilizes logic resources and introduces a delay when synthesized in a design. This insertion of checkers thus produces a noticeable impact on the design performance. The performance impact due to the VHDL OVL checkers is studied in the following section. The datasheet generated serves as a reference and is used for further research. When the performance impact due to the insertion of checkers needs to be studied, the reference data assists in estimating the resources used by the inserted checkers.

The study is performed by synthesizing and simulating each VHDL OVL checker on an FPGA in isolation. A single type of assertion checker is implemented for checking test data of widths ranging from 1 bit to 128 bits. The testbench consists of a number of checker instances connected in series to calculate the average performance metrics. The test data is passed through this series such that it is checked in each assertion module and then passed through to the next assertion module in series. The resulting data represents the average performance metrics for that assertion checker. The tests were performed for 4, 8 and 16 assertion checkers of each type connected in series. This

arrangement was used to reduce the skewing of the calculations and minimize the 
influence of other external factors. The generated datasheet for the VHDL OVL checkers is tabulated in Appendix B.

\subsection{Assertions in the proposed shifter design}

To improve its robustness and observability, assertion modules are inserted into the proposed shifter design. The primary function of the assertion modules is to verify the integrity of the data passing through the shifter and to perform $\mathrm{X} / \mathrm{Z}$ checks. This verifies that the data does not contain an $\mathrm{X}$ (unknown) or $\mathrm{Z}$ (high-impedance) value. Since the embedded logic is primarily a combinational-logic based (memory-less) design, there is little scope for insertion of assertion modules that check memory-based signals.

The shifter design utilizes three types of OVL assertion modules:

1. ovl_never_unknown: This assertion module is used as a constraint in the design. It performs an explicit $\mathrm{X} / \mathrm{Z}$ check to constrain the inputs and outputs of the design. The design uses an ovl_never_unknown checker each for the input $A$, the shift control $X$, the opcode $O$ and the output $B$. The size of the checker varies according to the size of the corresponding test expression.

2. ovl_next: The most-significant bit of the input data, $A_{n-1}$, is input to the shifter in every clock cycle of the shift process. The data on this pin remains constant for the complete shift cycle. This stability of $A_{n-1}$ is checked using the ovl_next module.

3. ovl_range: The value of the updated index $q$ is in the range,

$$
0 \leq q \leq \frac{n}{s}-1
$$

$q$ is calculated and used internally and thus cannot be measured through an output port. To check for any error related to the components calculating $q$, an 
ovl_range module is assigned to monitor $q$. Any $\mathrm{X} / \mathrm{Z}$ error or violation of the allowed range is reported by this assertion module. The ovl_range module can only be used when the test expression has a length of 2 bits or more. This checker is thus used only when the number of blocks to be processed is greater than 2. In the design under test, this checker cannot be used when implementing a 128-bit shifter using a 64-bit embedded logic or a 256-bit shifter using a 128-bit embedded logic.

The size and location of the assertion modules used in the 64-bit embedded logic and the 128-bit embedded logic are given in Table 7.2 and 7.3 respectively.

Table 7.2: OVL checker characteristics for the 64-bit embedded logic

\begin{tabular}{|c|c|c|c|c|c|c|}
\hline \multirow{2}{*}{$\begin{array}{c}\text { Effective } \\
\text { shifter } \\
\text { size }\end{array}$} & \multicolumn{5}{|c|}{ Test data width (bits) for assertion checkers used at specific ports } \\
\cline { 2 - 7 } & Input $\boldsymbol{A}$ & $\begin{array}{c}\text { Output } \\
\boldsymbol{B}\end{array}$ & $\begin{array}{c}\text { Shift } \\
\text { Control } \boldsymbol{X}\end{array}$ & Opcode $\boldsymbol{O}$ & $\begin{array}{c}\text { Updated } \\
\text { index } \boldsymbol{q}\end{array}$ & $\begin{array}{c}\text { Last bit } \\
\boldsymbol{A}_{(\boldsymbol{n}-\mathbf{1})}\end{array}$ \\
\hline $\mathbf{1 2 8}$ & 64 & 64 & 7 & 3 & 0 & 1 \\
\hline $\mathbf{2 5 6}$ & 64 & 64 & 8 & 3 & 2 & 1 \\
\hline $\mathbf{5 1 2}$ & 64 & 64 & 9 & 3 & 3 & 1 \\
\hline $\mathbf{1 0 2 4}$ & 64 & 64 & 10 & 3 & 4 & 1 \\
\hline
\end{tabular}

Table 7.3: OVL checker characteristics for the 128-bit embedded logic

\begin{tabular}{|c|c|c|c|c|c|c|}
\hline \multirow{2}{*}{\begin{tabular}{c} 
Effective $\begin{array}{c}\text { ovh__never_unknown } \\
\text { size }\end{array}$ \\
\cline { 2 - 7 }
\end{tabular}} & \multicolumn{5}{|c|}{ Test data width (bits) for assertion checkers used at specific ports } \\
\cline { 2 - 7 } & Input $\boldsymbol{A}$ & $\begin{array}{c}\text { Output } \\
\boldsymbol{B}\end{array}$ & $\begin{array}{c}\text { Shift } \\
\text { Control } \boldsymbol{X}\end{array}$ & Opcode $\boldsymbol{O}$ & $\begin{array}{c}\text { Updated } \\
\text { index } \boldsymbol{q}\end{array}$ & $\begin{array}{c}\text { Last bit } \\
\boldsymbol{A}_{(\boldsymbol{n} \text {-1 })}\end{array}$ \\
\hline $\mathbf{2 5 6}$ & 128 & 128 & 8 & 3 & 0 & 1 \\
\hline $\mathbf{5 1 2}$ & 128 & 128 & 9 & 3 & 2 & 1 \\
\hline $\mathbf{1 0 2 4}$ & 128 & 128 & 10 & 3 & 3 & 1 \\
\hline $\mathbf{2 0 4 8}$ & 128 & 128 & 11 & 3 & 4 & 1 \\
\hline
\end{tabular}




\subsection{Estimated resource overhead and performance reduction}

Using the data reference sheet in Appendix B, the performance of the assertion-based design can be estimated. The resource overhead and the delay due to the assertion checkers are added to the corresponding performance data in Section 6.5 for the proposed design. The estimated performance of the assertion-based design is given in Table 7.4. These estimations are cross-checked against the actual performance of the design when it is implemented.

Table 7.4: Estimated performance characteristics of an assertionbased shifter design

\begin{tabular}{|c|c|c|c|c|c|}
\hline $\begin{array}{l}\text { Shifter } \\
\text { Design }\end{array}$ & $\begin{array}{c}\text { Shifter } \\
\text { size } \\
\text { (bits) }\end{array}$ & $\begin{array}{c}\text { FPGA } \\
\text { Type }\end{array}$ & $\begin{array}{c}\text { I/O } \\
\text { utilization } \\
r / f \%\end{array}$ & $\begin{array}{l}\text { Estd. } \\
\text { Slices } \\
\text { Used }\end{array}$ & $\begin{array}{l}\text { Estd. } \\
\text { processing } \\
\text { delay (ns) }\end{array}$ \\
\hline \multirow{4}{*}{$\begin{array}{l}\text { Proposed } \\
\text { design: } \\
\text { 64-bit } \\
\text { auxiliary } \\
\text { shifter }\end{array}$} & 128 & $200 A$ & $62 \%$ & $51.3 \%$ & $13 \cdot 35$ \\
\hline & 256 & $200 A$ & $64 \%$ & $51.5 \%$ & 27.77 \\
\hline & 512 & $200 A$ & $64 \%$ & $52.6 \%$ & 63.71 \\
\hline & 1024 & $200 A$ & $65 \%$ & $56.7 \%$ & 120.18 \\
\hline \multirow{4}{*}{$\begin{array}{l}\text { Proposed } \\
\text { design: } \\
\text { 128-bit } \\
\text { auxiliary } \\
\text { shifter }\end{array}$} & 256 & $400 \mathrm{~A}$ & $91 \%$ & $57.1 \%$ & 17.42 \\
\hline & 512 & $400 \mathrm{~A}$ & $92 \%$ & $56.2 \%$ & 39.79 \\
\hline & 1024 & $400 \mathrm{~A}$ & $93 \%$ & $57.2 \%$ & 72.96 \\
\hline & 2048 & $400 \mathrm{~A}$ & $93 \%$ & $58.3 \%$ & 163.40 \\
\hline
\end{tabular}




\section{Chapter 8}

\section{Alleviating Performance Overhead Due to Assertions}

The insertion of the assertion checkers increases the design transparency and controllability during the simulation phase. However, it also leads to a logic resource overhead and an increase in delay. To alleviate the performance impact, a novel approach to assertion-based FPGA design is proposed.

The shifter design is implemented with the required assertion modules. However, the assertion modules are partitioned into a separate logical block on the FPGA floorplan. The test data is routed to the assertion modules' block where it is checked for the corresponding constraints and errors.

The proposed approach permits the creation of the shifter design with a placement similar to a non assertion-based approach. The proposed design still uses assertion modules and thus allows the use of $\mathrm{ABV}$ to test it. When the verification process is complete, the FPGA is reconfigured to remove the assertion modules. However, the reconfiguration is performed without changing the placement of the shifter design. The FPGA is reconfigured only to remove the logical block containing the assertion modules. This approach results in a minimal reconfigu ration runtime.

The proposed design, however, possesses certain characteristics:

1. When the design is first implemented with the assertion modules' block, the total design delay is higher compared to a conventional assertion-based design. The 
partitioning of the assertion modules increases the data path that the test data travels, thus increasing the delay.

2. The placement of the shifter design is relatively inefficient compared to a nonassertion based shifter design. This is due to the addition of the assertion modules in the design. This increases the design delay.

3. The runtime required for reconfiguration is lower compared to the initial design placement and timing process. This is because the design placement of the shifter is preserved when the assertion modules are removed.

\subsection{Partitioning and component removal in Xilinx ISE [25]}

Partitions in Xilinx ISE help optimize the implementation process by preserving unchanged implemented portions of the design. If the HDL, timing, physical constraints and implementation options of partition are unchanged, the implementation tools will use a "copy-and-paste" process to guarantee that the implementation data for that Partition is preserved.

The preservation level for a Partition can be set to one of the following:

- $\quad$ Routing

- Placement

- Synthesis

- Inherit

The preservation level can be changed from the default level of "routing" for each partition. The amount of design data preserved will decrease as the preservation level is changed from "routing" to "placement" to "synthesis". 


\subsubsection{Routing}

"Routing" preserves the data of the partition through routing. This level of preservation gives the implementation tools the least amount of flexibility to meet the timing or implementation objectives, but provides the highest degree of preservation. The following implementation data is always preserved at the "routing" preservation level:

1. Synthesized netlist and information

2. Placement information

3. Routing information

\subsubsection{Placement}

"Placement" preserves the data of the partition through placement. The following implementation data is always preserved for the "placement" preservation level:

1. Synthesized netlist and information

2. Placement information

3. Some Routing information, possibly all routing information

\subsubsection{Synthesis}

"Synthesis" only preserves the synthesis netlist of the design. The following implementation data is always preserved at the "synthesis" preservation level:

- Synthesized netlist and information

- Some placement information, possibly all placement information

- Some routing information, possibly all routing information. 


\subsubsection{Inherit}

The "inherit" preservation level sets the "preserve" attribute to the same level as the parent partition. The default setting for all child partitions is "inherit".

\subsection{Using Xilinx Partitions in the design under study}

The proposed approach partitions the assertion-based shifter design into two logical blocks. The shifter design is placed in one block while the inserted assertion modules are partitioned into the other block. The partitioning process is performed at the HDL level. The shifter design and the assertion modules are defined in separate entities and are interfaced through the test data that is to be monitored by the assertion checkers. This test data is passed to the assertion modules by the shifter design.

This HDL design is implemented on the FPGA. Following this, the preservation level of the shifter design is set to "placement" and the assertion modules removed. The "placement" preservation level preserves the placement of the shifter design when reconfiguring the FPGA. The performance characteristics of the reconfigured shifter design are then generated.

\subsection{Experimental results and analysis}

\subsubsection{Assertion-based design}

The performance characteristics of the design described in Section 8.2 are tabulated in Table 8.1. Table 8.2 compares the percentage increase in the resource overhead and design delay of the proposed assertion-based design over the assertion free design implemented in Section 6.5. The results show that the assertion-based design has an average increase of $14.8 \%$ in the number of slices utilized. Table 8.3 further indicates 
that the actual overhead slice usage of the design is an average of $7.5 \%$ higher than the estimated increase calculated in Section 7.9.

The design delay in the assertion-based design also increases by an average of $40 \%$ while the estimate of the average delay increase is $18.3 \%$ lower than the actual design delay.

The variation in the performance from the mathematical estimation can be attributed a number of factors including,

1. The reference datasheet utilizes identical assertion checkers arranged in series on a single FPGA. The checkers implemented are instances of the same HDL design. This may cause sharing of resources during implementation to optimize the design. This leads to a reduction in the number of slices actually used. The assertion modules implemented in the assertion-based design are of different types and sizes. The resource sharing ability of th is design is reduced in this case. This leads to the skewing of the data.

2. The proposed design partitions the assertion modules into a separate logic block. Due to this, the data to be checked travels a longer datapath to the assertion checker. Each test data to be monitored utilizes a different datapath. The comparatively inefficient routing of the signals leads to an increase in the longest path delay of the design, thus increasing the design delay.

3. As the size of the design increases, the placement efficiency of the assertion modules decreases. This further degrades the performance. 
Table 8.1: Performance characteristics of various assertion-based designs

\begin{tabular}{|c|c|c|c|c|c|c|}
\hline $\begin{array}{l}\text { Shifter } \\
\text { Design }\end{array}$ & $\begin{array}{c}\text { Shifter } \\
\text { size } \\
\text { (bits) } \\
\end{array}$ & $\begin{array}{l}\text { FPGA } \\
\text { Type }\end{array}$ & $\begin{array}{c}\text { I/O } \\
\text { utilization } \\
\text { r/f\% } \\
\end{array}$ & $\begin{array}{l}\text { Slices } \\
\text { Used }\end{array}$ & $\begin{array}{c}\text { Total } \\
\text { processing } \\
\text { delay (ns) }\end{array}$ & $\begin{array}{c}\text { Configuration } \\
\text { Time (s) }\end{array}$ \\
\hline \multirow{4}{*}{$\begin{array}{l}\text { Proposed } \\
\text { design: } \\
\text { 64-bit } \\
\text { auxiliary } \\
\text { shifter }\end{array}$} & 128 & $200 \mathrm{~A}$ & $62 \%$ & $61 \%$ & 16.21 & 183 \\
\hline & 256 & $200 A$ & $64 \%$ & $66 \%$ & 33.81 & 231 \\
\hline & 512 & $200 A$ & $64 \%$ & $67 \%$ & 73.22 & 280 \\
\hline & 1024 & $400 A$ & $65 \%$ & $67 \%$ & 152.96 & 379 \\
\hline \multirow{4}{*}{$\begin{array}{l}\text { Proposed } \\
\text { design: } \\
\text { 128-bit } \\
\text { auxiliary } \\
\text { shifter }\end{array}$} & 256 & $400 \mathrm{~A}$ & $91 \%$ & $59 \%$ & 21.47 & 212 \\
\hline & 512 & $400 \mathrm{~A}$ & $92 \%$ & $59 \%$ & 41.87 & 511 \\
\hline & 1024 & $400 \mathrm{~A}$ & $93 \%$ & $60 \%$ & 90.77 & 627 \\
\hline & 2048 & $400 \mathrm{~A}$ & $93 \%$ & $61 \%$ & 180.16 & 1235 \\
\hline
\end{tabular}

Table 8.2: Performance degradation in an assertion-based design compared to an assertion-free design

\begin{tabular}{|c|r|c|c|c|}
\hline $\begin{array}{c}\text { Shifter } \\
\text { Design }\end{array}$ & $\begin{array}{c}\text { Shifter } \\
\text { size } \\
\text { (bits) }\end{array}$ & $\begin{array}{c}\text { I/O } \\
\text { utilization } \\
\boldsymbol{r} / \mathbf{f} \%\end{array}$ & $\begin{array}{c}\text { Slices } \\
\text { Used }\end{array}$ & $\begin{array}{c}\text { Total } \\
\text { processing } \\
\text { delay (ns) }\end{array}$ \\
\hline $\begin{array}{c}\text { Proposed } \\
\text { design: } \\
\text { 64-bit }\end{array}$ & 128 & $6 \%$ & $17 \%$ & $41 \%$ \\
\cline { 2 - 5 } $\begin{array}{c}\text { auxiliary } \\
\text { shifter }\end{array}$ & 256 & $8 \%$ & $22 \%$ & $45 \%$ \\
\cline { 2 - 5 } & 512 & $7 \%$ & $22 \%$ & $36 \%$ \\
\hline $\begin{array}{c}\text { Proposed } \\
\text { design: }\end{array}$ & 256 & $8 \%$ & $18 \%$ & $55 \%$ \\
\cline { 2 - 5 } $\begin{array}{c}\text { 128-bit } \\
\text { auxiliary } \\
\text { shifter }\end{array}$ & 512 & $6 \%$ & $9 \%$ & $42 \%$ \\
\cline { 2 - 5 } & 2048 & $6 \%$ & $10 \%$ & $48 \%$ \\
\hline
\end{tabular}

Table 8.3: Percentage error in estimating the performance metrics of an assertion-based design

\begin{tabular}{|l|r|c|c|}
\hline \multirow{2}{*}{$\begin{array}{l}\text { Shifter } \\
\text { Design }\end{array}$} & $\begin{array}{c}\text { Shifter } \\
\text { size } \\
\text { (bits) }\end{array}$ & $\begin{array}{l}\text { Slices } \\
\text { Used }\end{array}$ & $\begin{array}{c}\text { Total } \\
\text { processing } \\
\text { delay (ns) }\end{array}$ \\
\hline $\begin{array}{l}\text { Proposed } \\
\text { design: }\end{array}$ & 128 & $10 \%$ & $21 \%$ \\
\cline { 2 - 4 } $\begin{array}{l}\text { 64-bit } \\
\text { auxiliary } \\
\text { shifter }\end{array}$ & 256 & $14 \%$ & $22 \%$ \\
\cline { 2 - 4 } & 512 & $14 \%$ & $15 \%$ \\
\hline $\begin{array}{l}\text { Proposed } \\
\text { design: }\end{array}$ & 256 & $11 \%$ & $27 \%$ \\
\cline { 2 - 4 } $\begin{array}{l}\text { 128-bit } \\
\text { auxiliary } \\
\text { shifter }\end{array}$ & 1024 & $3 \%$ & $23 \%$ \\
\cline { 2 - 4 } & 2048 & $3 \%$ & $5 \%$ \\
\hline
\end{tabular}




\subsubsection{Re-configured assertion-free design}

The assertion checkers can be removed from the design once the verification process is completed. This is performed by reconfiguring the FPGA to remove the assertion modules while preserving the placement of the shifter design. The performance gains from the reconfiguration are shown in Table 8.4.

The removal of the assertion modules results in a constant reduction of $8 \%$ in the slices used for the shifters implemented using 64-bit and 128-bit embedded logic. The reconfiguration also leads to a $32 \%$ reduction in the total processing delay. Table 8.4 also shows the reconfiguration runtime required to remove the assertion checkers and reconfigure the FPGA. The use of Partitions and the "Placement" preservation level for the embedded shifter limits the average reconfiguration runtime to a modest $31 \%$ of the initial configuration time.

The reconfigured design is slightly less efficient and slower than the assertionfree design implemented in Section 6.5. However, the use of assertions significantly decreases the time required for verification. This ultimately leads to a shorter time to market of the design.

Table 8.4: Performance gain through assertion removal

\begin{tabular}{|l|c|c|c|c|}
\hline \multirow{2}{*}{$\begin{array}{l}\text { Shifter } \\
\text { Design }\end{array}$} & $\begin{array}{c}\text { Shifter } \\
\text { size } \\
\text { (bits) }\end{array}$ & $\begin{array}{c}\text { Slices } \\
\text { Reduction } \\
\text { \% }\end{array}$ & $\begin{array}{c}\text { Processing } \\
\text { delay } \\
\text { reduction\% }\end{array}$ & $\begin{array}{c}\text { Reconfiguration } \\
\text { time \% }\end{array}$ \\
\hline $\begin{array}{l}\text { Proposed } \\
\text { design: }\end{array}$ & 128 & $8 \%$ & $17.4 \%$ & $30.6 \%$ \\
\cline { 2 - 5 } $\begin{array}{l}\text { 64-bit } \\
\text { auxiliary } \\
\text { shifter }\end{array}$ & $\mathbf{2 5 6}$ & $8 \%$ & $24.0 \%$ & $31.2 \%$ \\
\cline { 2 - 5 } & 1024 & $8 \%$ & $22.2 \%$ & $32.9 \%$ \\
\hline $\begin{array}{l}\text { Proposed } \\
\text { design: }\end{array}$ & 256 & $7 \%$ & $30.5 \%$ & $31.1 \%$ \\
\cline { 2 - 5 } $\begin{array}{l}\text { 128-bit } \\
\text { auxiliary } \\
\text { shifter }\end{array}$ & 512 & $8 \%$ & $13.8 \%$ & $32.1 \%$ \\
\cline { 2 - 5 } & 1024 & $8 \%$ & $32.2 \%$ & $31.3 \%$ \\
\hline
\end{tabular}


The choice of approach for the design implementation is based on the design requirements. A design that has short time-to-market can be implemented using assertion-based design. This reduces the time needed for design verification. However, when the design performance is the primary constraint, the use of other verification methodologies may be preferable. 


\section{Chapter 9}

\section{Conclusion and Future Work}

\subsection{Conclusion}

Conventional shifter designs implemented on an FPGA are limited by its size due to I/O pin constraints. A novel approach has been proposed in this thesis that alleviates this major bottleneck and allows implementation of large shifters. It uses combinational and sequential hybrid design with varying complexities of the embedded logic. The algorithm has been translated into an HDL design which has been optimized for use on FPGAs. The design is implemented on the Spartan-3A FPGAs. The proposed design is suitable for real-time applications due to improved performance, low chip area utilization, and fewer I/O pins. The proposed approach can be seamlessly extended for multi-chip FPGA designs.

The use of OVL assertion checkers in the design is proposed to allow ease of verification. Assertion checkers are placed at strategic points in the design and used as constraints and improve transparency of the design. The use of these assertion checkers leads to an increase in the logic resources utilized and the total delay of the design. These problems are addressed through the use of a novel approach to assertion-based design. The proposed approach reduces the resources utilized and the performance delay. The low reconfiguration runtime of the approach allows its implementation in practical scenarios.

\subsection{Future work}

The objective of the proposed design was to alleviate the I/O bottleneck of FPGA-based design with a minimal degradation in performance. Further research work on this topic would focus on 
the modification of the design to improve the design performance. Improving the HDL design of the proposed approach would be the first step to this optimization. The largest logical block in the HDL design is the ROM implemented in the combinational logic of the shifter. An optimized version of this design would implement this logical block in a more efficient manner.

The efficiency of the placement and routing of any design is dependent on the skill of the designer. Apart from the use of a faster FPGA, a hardware designer skilled in placement methodologies could implement the design with more favorable performance results. The use of a newer software versions and more efficient placement algorithms can lead to a better performance of the design.

Assertion-based verification is still in the early stages of development. While the use of assertions is not a recent idea, the practical use of assertions in hardware verification to improve productivity has gained importance only in recent years. The creation of assertion standards has accelerated the use of assertions amongst the verification community.

The thesis discussed the placement of assertion checkers in the design and the resource and performance overhead due to the insertion of assertion checkers. The next logical step to further the research is to document the verification process of the design through a combination of formal verification and assertion based verification. The formal verification of the design requires the development of a mathematical process to formally verify the design.

The assertions introduced in the design act as constraints or provide a monitor to observe the functioning of an internal component of the design. The number of assertions used is dependent on the final objective of the exercise. The use of a large number of assertions increases the transparency of the design, but leads to a large number of outputs. The design would then require an I/O optimization for the use of these assertions. 


\section{References}

[1] R. S. Lim, “A Barrel Switch Design,” Computer Design, pp. 76-78, August 1972.

[2] M. R. Pillmeier, "Barrel Shifter Design, Optimization, and Analysis," Master's thesis, Lehigh University, January 2002.

[3] K. P. Acken, M. J. Irwin, and R. M. Owens, "Power Comparisons for Barrel Shifters," Proc. of the Int. Symp. on Low Power Electronics and Design, pp. 209-212, 1996.

[4] G. M. Tharakan and S. M. Kang, "A New Design of a Fast Barrel Switch Network," IEEE Journal of Solid-State Circuits, vol. 28, pp. 217-221, February 1992.

[5] S.-J. Yih, M. Cheng, and W.-S. Feng, "Multilevel Barrel Shifter for CORDIC Design," Electronics Letters, vol. 32, pp. 1178-1179, June 1996.

[6] P. A. Beerel, S. Kim, P.-C. Yeh, and K. Kim, "Statistically Optimized Asynchronous Barrel Shifters for Variable Length Codecs," Proc. of the Int. Symp. on Low Power Electronics and Design, pp. 261-263, 1999.

[7] M. R. Pillmeier, M. J. Schulte, and E. G. Walters III, "Design Alternatives for Barrel Shifters," Proc. of SPIE: Advanced Signal Processing Algorithms, Architectures, and Implementations, vol. 4791, pp. 436-447, Seattle, Washington, July 2002.

[8] H. D. Foster, A. C. Krolnik, and D. J. Lacey, Assertion-Based Design, 2nd ed. Kluwer Academic Publishers, 2003.

[9] K. C. Chen, "Assertion-based verification for SoC designs," Proc. of the 5th International ASIC Conference, vol. 1, pp. 12-15, October 2003.

[10] B. Meyer, "Applying design by contract," IEEE Computer, vol. 25, pp. 40-51, 1992.

[11] P. Yeung, K. Larsen, "Practical Assertion-based Formal Verification for SoC Designs," Proc. International Symposium on System-on-Chip, pp. 58-61, 15-17 November 2005.

[12] J. M. Voas, K. W. Miller, "Putting assertions in their place," Proc. of International Symposium on Software Reliability Engineering, pp. 152-157, 1994.

[13] M. Riazati, S. Mohammadi, A. Afzali-Kusha, and Z. Navabi, "Improved Assertion Lifetime via Assertion-Based Testing Methodology," International Conference on Microelectronics, pp. 48-51, 16-19 December 2006.

[14] Novas Software Inc., "SystemVerilog: An EDA Vendor Perspective," The 12th EDA Interoperability Developers' Forum, October 2003.

[15] J. Bhasker, A VHDL Primer, 3rd ed. Pearson Education, 2005.

[16] W. Wolf, FPGA-Based System Design, 1st ed. Prentice Hall, 2004. 
[17] S. Brown, J. Rose, "Architecture of FPGAs and CPLDs: A Tutorial," IEEE Design and Test of Computers, vol. 13, no. 2, pp. 42-57, 1996.

[18] L. Berger, A. Greiner, and E. P. Lopes, "A Consistent Approach in Logic Synthesis for FPGA Architectures," Proceedings of the International Conference on ASIC, pp. 104107, Pekin, October 1994.

[19] L. Scheffer, L. Lavagno, Electronic Design Automation For Integrated Circuits Handbook, 1st ed. CRC Press, 2006.

[20] H. Foster, K. Larsen, M. Turpin, "Introduction to the New Accellera Open Verification Library." [Online]. Available: http://www.eda.org/ovl/pages/pdfs/dvcono6 foster.pdf

[14] Novas Software Inc, SystemVerilog: An EDA Vendor Perspective, in The 12th EDA Interoperability Developers' Forum, October 2003

[21] R. Stolzman, "Understanding Assertion-Based Verification.” [Online]. Available: http://www.edadesignline.com/showArticle.jhtml?articleID=192200468

[22] J. Horgan, "Assertion Based Verification." [Online]. Available: http://www10.edacafe.com/nbc/articles/view weekly.php?articleid=209195\&page no= $\underline{1}$

[23] Accellera Organization Inc. [Online]. Available: http://www.accellera.org/

[24] Accellera Organization Inc., "Accelera Standard OVL v2.o - Library Reference Manual", June 2007.

[25] C. Zeh, "Incremental Design Reuse with Partitions" [Online]. Available: http://www.xilinx.com/support/documentation/application notes/xapp918.pdf

[26] A.Cosoroaba, F. Rivoallon, "Achieving Higher System Performance with the Virtex-5 Family of FPGAs." [Online]. Available: http://www.xilinx.com/bvdocs/whitepapers/wp245.pdf

[27] Xilinx, Inc., [Online]. Available: http://www.xilinx.com/

[28] Altera Corp., [Online]. Available: http://www.altera.com/

[29] Lattice Semiconductor Corp., [Online]. Available: http://www.latticesemi.com/

[30] Actel Corp., [Online]. Available: http://www.actel.com/

[31] Atmel Corp., [Online]. Available: http://www.atmel.com/

[32] Z. A. Syed, A. Noore, "Performance Optimization to Alleviate I/O Constraints in Designing Large FPGA Shifters," IEICE Electronics Express, vol. 5, no. 1, pp. 29-34, 2008. 


\section{Appendix}

\section{Appendix A}

\section{A.1 The VHDL OVL library}

The accellera_ovl_hdl [24] library contains VHDL implementations of the OVL checkers. The v2.1 of the VHDL OVL library released in Sep 2007 implements 10 of the most commonly used OVL checkers in verification. The remaining 21 checkers will be addressed in later versions of the VHDL OVL library. The functions of the 10 VHDL OVL checkers are described in Table A.1. The VHDL OVL components are compatible with the Verilog OVL versions. However, the VHDL components include an additional generic named controls that provides global configuration of the library. The VHDL OVL library is synthesizable and its components support both std_logic/std_logic_vector and std_ulogic/std_ulogic_vector port types.

\section{A.1.1 The ovl_ctrl_record type}

The global library configuration is controlled by an ovl_ctrl_record constant assigned to the controls generic on every checker instance. This constant is defined in every design package so that the global variables can be controlled from a single place.

The ovl_ctrl_record type is divided into three groups:

1. Elements that are of the ovl_ctrl type and can be assigned OVL_ON or OVL_OFF values. These elements mainly control the generate statements used in the checkers. 
2. User-configurable values that control the message printing and how long the simulation should continue after a fatal assertion occurs.

3. Default values of the generics that are common to all checkers.

The ovl_ctrl_record type variable that is used to control the design package for the design under study is given in the Appendix.

Table A.1: Description of the VHDL OVL checkers [24]

\begin{tabular}{|c|c|}
\hline Assertion Statement & Description \\
\hline ovl_always & $\begin{array}{l}\text { Checks the single-bit expression test_expr at each rising } \\
\text { edge of clk to verify whether it evaluates to TRUE. }\end{array}$ \\
\hline ovl_cycle_sequence & $\begin{array}{l}\text { Checks the expression event_sequence at the rising edges } \\
\text { of clk to identify whether or not the bits in event_sequence } \\
\text { assert sequentially on successive rising edges of clk. }\end{array}$ \\
\hline ovl_never & $\begin{array}{l}\text { Checks the single-bit expression test_expr at each rising } \\
\text { edge of clk to verify the expression does not evaluate to } \\
\text { TRUE. }\end{array}$ \\
\hline ovl_one_hot & $\begin{array}{l}\text { Checks the expression test_expr at each rising edge of clk } \\
\text { to verify the expression evaluates to a one-hot value. A } \\
\text { one-hot value has exactly one bit set to } 1 \text {. }\end{array}$ \\
\hline ovl_range & $\begin{array}{l}\text { Checks the expression test_expr at each rising edge of clk } \\
\text { to verify the expression falls in the range from min to } \max \text {, } \\
\text { inclusive. The assertion fails if test_expr }<\min \text { or } \max < \\
\text { test_expr. }\end{array}$ \\
\hline ovl_implication & $\begin{array}{l}\text { If antecedent_expr holds then consequent_expr must hold } \\
\text { in the same cyle }\end{array}$ \\
\hline ovl_never_unknown & $\begin{array}{l}\text { test_expr must never be an unknown value, just boolean } 0 \\
\text { or } 1\end{array}$ \\
\hline ovl_never_unknown_async & $\begin{array}{l}\text { test_expr must never be an unknown value } \\
\text { asynchronously, it must remain boolean o or } 1\end{array}$ \\
\hline ovl_next & $\begin{array}{l}\text { test_expr must hold num_cks cycles after start_event } \\
\text { holds }\end{array}$ \\
\hline ovl_zero_one_hot & $\begin{array}{l}\text { test_expr must be one-hot or zero, i.e. at most one bit set } \\
\text { high }\end{array}$ \\
\hline
\end{tabular}

\section{A.1.2 Synthesizing the VHDL OVL library}

All the code in the OVL VHDL library is completely synthesizable except the path_name attribute in the architectures and the std_ovl_procs.vhd file. This issue is fixed by 
modifying the architecture bodies to set the path string constants to "“ and using the std_ovl_procs_syn.vhd file in the std_ovl directory. 


\section{Appendix B}

\section{VHDL OVL Checker Performance Data}

This appendix lists the performance characteristics of the VHDL OVL checkers. The data is generated using the procedure described in Section 7.8. Each table lists the resource utilization and delay characteristics of each checker. The size of the test data varies from 1 bit to 128 bits for each checker. To calculate an average value, 4, 8, and 16 checkers are implemented for every test data width.

\begin{tabular}{|c|c|c|c|c|c|}
\hline \multicolumn{6}{|c|}{ Assert never unknown - Slices Used } \\
\hline \multirow{2}{*}{$\begin{array}{l}\text { Number of } \\
\text { checkers }\end{array}$} & \multicolumn{5}{|c|}{ Test Data Width (bits) } \\
\hline & 1 & 4 & 16 & 64 & 128 \\
\hline 4 & 5 & 14 & 56 & 242 & 486 \\
\hline 8 & 9 & 29 & 118 & 510 & 946 \\
\hline 16 & 14 & 62 & 213 & 1014 & 1920 \\
\hline \multicolumn{6}{|c|}{ Assert never unknown - Total delay (ns) } \\
\hline \multirow{2}{*}{$\begin{array}{l}\text { Number of } \\
\text { checkers }\end{array}$} & \multicolumn{5}{|c|}{ Test Data Width (bits) } \\
\hline & 1 & 4 & 16 & 64 & 128 \\
\hline 4 & 0.29 & 0.66 & 0.98 & 0.86 & 1.21 \\
\hline 8 & 0.57 & 1.44 & 1.66 & 1.19 & 1.59 \\
\hline 16 & 1.25 & 2.30 & 2.21 & 1.63 & 2.19 \\
\hline
\end{tabular}

\begin{tabular}{|r|rrrrr|}
\hline \multicolumn{6}{|c|}{ Assert always - Slices Used } \\
\hline Number of \\
checkers & 1 & 4 & 16 & 64 & 128 \\
\cline { 2 - 6 } & 3 & 11 & 43 & 157 & 335 \\
8 & 5 & 17 & 94 & 298 & 641 \\
16 & 10 & 37 & 156 & 514 & 1302 \\
\hline \multicolumn{5}{|c|}{ Assert always - Total delay (ns) } \\
\hline Number of & \multicolumn{5}{|c|}{ Test Data Width (bits) } \\
\cline { 2 - 6 } checkers & 1 & 4 & 16 & 64 & 128 \\
\hline 4 & 0.95 & 0.73 & 0.91 & 1.20 & 2.24 \\
8 & 1.66 & 1.55 & 1.52 & 1.59 & 2.50 \\
16 & 1.70 & 1.53 & 2.02 & 2.61 & 2.54 \\
\hline
\end{tabular}

\begin{tabular}{|r|rrrrr|}
\hline \multicolumn{6}{|c|}{ Assert range - Slices Used } \\
\hline \multirow{2}{*}{$\begin{array}{c}\text { Number of } \\
\text { checkers }\end{array}$} & 1 & 4 & 16 & 64 & 128 \\
\cline { 2 - 6 } & 10 & 35 & 134 & 542 & 1002 \\
8 & 19 & 78 & 273 & 1031 & 2046 \\
16 & 39 & 139 & 522 & 2079 & 4102 \\
\hline \multicolumn{5}{|c|}{ Assert range - Total delay (ns) } \\
\hline Number of & \multicolumn{5}{|c|}{ Test Data Width (bits) } \\
\cline { 2 - 6 } checkers & 1 & 4 & 16 & 64 & 128 \\
\hline 4 & 0.40 & 0.72 & 0.93 & 1.46 & 1.94 \\
8 & 1.90 & 1.62 & 2.56 & 1.79 & 2.78 \\
16 & 2.10 & 2.82 & 2.06 & 2.27 & 2.01 \\
\hline
\end{tabular}

\begin{tabular}{|r|rrrrr|}
\hline \multicolumn{5}{|c|}{ Assert cycle sequence - Slices Used } \\
\hline $\begin{array}{r}\text { Number of } \\
\text { checkers }\end{array}$ & 1 & 4 & 16 & 64 & 128 \\
\cline { 2 - 6 } & 6 & 29 & 145 & 401 & 870 \\
8 & 11 & 63 & 413 & 914 & 1610 \\
16 & 25 & 136 & 846 & 1649 & 3132 \\
\hline \multicolumn{5}{|c|}{ Test Data Width (bits) } \\
\hline \multicolumn{5}{|c|}{ Assert cycle sequence - Total delay (ns) } \\
\hline Number of & 1 & 4 & 16 & 64 & 128 \\
\cline { 2 - 6 } checkers & 1.49 & 0.83 & 0.80 & 1.83 & 1.72 \\
8 & 1.62 & 1.36 & 1.08 & 1.70 & 2.34 \\
16 & 1.83 & 2.25 & 2.46 & 2.31 & 2.70 \\
\hline
\end{tabular}




\begin{tabular}{|r|rrrrr|}
\hline \multicolumn{6}{|c|}{ Assert next - Slices Used } \\
\hline Number of $\begin{array}{r}\text { Test Data Width (bits) } \\
\text { checkers }\end{array}$ & 1 & 4 & 16 & 64 & 128 \\
\cline { 2 - 6 } & 13 & 52 & 195 & 772 & 1540 \\
8 & 22 & 102 & 372 & 1488 & 3055 \\
16 & 45 & 199 & 734 & 3013 & 6202 \\
\hline \multicolumn{5}{|c|}{ Assert next - Total delay (ns) } \\
\hline Number of & \multicolumn{5}{|c|}{ Test Data Width (bits) } \\
\cline { 2 - 6 } checkers & 1 & 4 & 16 & 64 & 128 \\
\hline 4 & 1.14 & 1.05 & 1.76 & 2.41 & 2.58 \\
8 & 1.11 & 1.68 & 2.93 & 2.26 & 3.09 \\
16 & 2.58 & 2.15 & 2.29 & 2.96 & 3.93 \\
\hline
\end{tabular}

\begin{tabular}{|r|rrrrr|}
\hline \multicolumn{6}{|c|}{ Assert never - Slices Used } \\
\hline Number of \\
checkers & 1 & 4 & 16 & 64 & 128 \\
\cline { 2 - 6 } 4 & 5 & 13 & 53 & 210 & 492 \\
8 & 8 & 31 & 109 & 489 & 889 \\
16 & 13 & 55 & 202 & 997 & 1834 \\
\hline \multicolumn{6}{|c|}{ Assert never - Total delay (ns) } \\
\hline Number of & \multicolumn{5}{|c|}{ Test Data Width (bits) } \\
\cline { 2 - 6 } checkers & 1 & 4 & 16 & 64 & 128 \\
\hline 4 & 0.75 & 1.24 & 1.21 & 1.44 & 1.41 \\
8 & 0.55 & 0.96 & 1.73 & 2.20 & 2.13 \\
16 & 1.65 & 1.32 & 2.36 & 2.71 & 2.98 \\
\hline
\end{tabular}

\begin{tabular}{|r|rrrrr|}
\hline \multicolumn{6}{|c|}{ Assert zero one hot - Slices Used } \\
\hline Number of \\
\cline { 2 - 6 } checkers & 1 & 4 & 16 & 64 & 128 \\
\hline 4 & 8 & 35 & 157 & 438 & 931 \\
8 & 12 & 64 & 457 & 922 & 1591 \\
16 & 27 & 166 & 839 & 1576 & 2855 \\
\hline \multicolumn{5}{|c|}{ Test Data Width (bits) } \\
\hline Number of & \multicolumn{5}{|c|}{ Test Data Width (bits) } \\
\cline { 2 - 6 } checkers & 1 & 4 & 16 & 64 & 128 \\
\hline 4 & 1.28 & 1.17 & 1.16 & 1.90 & 1.99 \\
8 & 1.62 & 1.85 & 1.31 & 2.13 & 2.37 \\
16 & 1.67 & 1.80 & 2.63 & 2.81 & 2.66 \\
\hline
\end{tabular}

\begin{tabular}{|r|rrrrr|}
\hline \multicolumn{6}{|c|}{ Assert one hot - Slices Used } \\
\hline Number of $\begin{array}{r}\text { Test Data Width (bits) } \\
\text { checkers }\end{array}$ & 1 & 4 & 16 & 64 & 128 \\
\cline { 2 - 6 } 4 & 7 & 28 & 149 & 422 & 910 \\
8 & 10 & 57 & 434 & 902 & 1553 \\
16 & 22 & 143 & 823 & 1567 & 2831 \\
\hline \multicolumn{6}{|c|}{ Assert one hot - Total delay (ns) } \\
\hline Number of & \multicolumn{5}{|c|}{ Test Data Width (bits) } \\
\cline { 2 - 6 } checkers & 1 & 4 & 16 & 64 & 128 \\
\hline 4 & 0.21 & 0.33 & 1.18 & 2.16 & 1.47 \\
8 & 1.10 & 1.60 & 1.66 & 2.97 & 2.50 \\
16 & 1.59 & 1.98 & 2.22 & 2.60 & 3.66 \\
\hline
\end{tabular}

\begin{tabular}{|r|rrrrr|}
\hline \multicolumn{6}{|c|}{ Assert implication - Slices Used } \\
\hline Number of $\begin{array}{r}\text { Test Data Width (bits) } \\
\text { checkers }\end{array}$ & 1 & 4 & 16 & 64 & 128 \\
\cline { 2 - 6 } & 15 & 37 & 192 & 712 & 1376 \\
8 & 20 & 66 & 344 & 1213 & 2668 \\
16 & 36 & 187 & 751 & 2787 & 4532 \\
\hline \multicolumn{5}{|c|}{ Assert implication - Total delay (ns) } \\
\hline Number of & \multicolumn{5}{|c|}{ Test Data Width (bits) } \\
\cline { 2 - 6 } checkers & 1 & 4 & 16 & 64 & 128 \\
\hline \multirow{2}{*}{4} & 0.86 & 1.38 & 1.65 & 2.00 & 2.63 \\
8 & 1.13 & 1.74 & 2.23 & 2.76 & 2.95 \\
16 & 1.77 & 1.93 & 2.64 & 3.01 & 3.70 \\
\hline
\end{tabular}

\begin{tabular}{|c|c|c|c|c|c|}
\hline \multicolumn{6}{|c|}{ Assert never unknown async - Slices Used } \\
\hline \multirow{2}{*}{$\begin{array}{l}\text { Number of } \\
\text { checkers }\end{array}$} & \multicolumn{5}{|c|}{ Test Data Width (bits) } \\
\hline & 1 & 4 & 16 & 64 & 128 \\
\hline 4 & 5 & 14 & 56 & 242 & 486 \\
\hline 8 & 9 & 29 & 118 & 510 & 946 \\
\hline 16 & 14 & 62 & 213 & 1014 & 1920 \\
\hline \multicolumn{6}{|c|}{ Assert never unknown async - Total delay (ns) } \\
\hline \multirow{2}{*}{$\begin{array}{c}\text { Number of } \\
\text { checkers }\end{array}$} & \multicolumn{5}{|c|}{ Test Data Width (bits) } \\
\hline & 1 & 4 & 16 & 64 & 128 \\
\hline 4 & 0.68 & 1.22 & 1.41 & 1.55 & 1.73 \\
\hline 8 & 0.48 & 1.41 & 2.80 & 2.44 & 2.03 \\
\hline 16 & 1.07 & 1.65 & 2.96 & 2.33 & 2.61 \\
\hline
\end{tabular}

